\title{
ON AN ASYMPTOTIC MODEL FOR FREE BOUNDARY DARCY FLOW IN POROUS MEDIA*
}

\author{
RAFAEL GRANERO-BELINCHÓN ${ }^{\dagger}$ AND STEFANO SCROBOGNA S $^{\ddagger}$
}

\begin{abstract}
We provide a rigorous mathematical study of an asymptotic model describing Darcy flow with free boundary in a small amplitude/large wavelength approximation. In particular, we prove several well-posedness results in critical spaces. Furthermore, we also study how the solution decays towards the flat equilibrium.
\end{abstract}

Key words. Muskat problem, moving interfaces, free boundary problems

AMS subject classifications. 35455, 35B41, 92C17

DOI. $10.1137 / 19 \mathrm{M} 126623 \mathrm{X}$

1. Introduction. Since the pioneering works of Boussinesq [8], the derivation and study of asymptotic models for free boundary flows (usually, the water waves problem) is a hot research area (the interested reader can refer to [43]).

In this paper we study an asymptotic model for the intrusion of water into oil sand. This is known as the Muskat problem [44]. The study of the (full) Muskat problem has received a lot of attention in the last years with several very recent advancements on the problem (cf. $[1,2,4,5,31,45]$ ), and, as a consequence, there is a large literature available (we refer to [36] for a recent survey of the available results). More precisely, this work considers the following one-dimensional equation:

$$
\left\{\begin{array}{l}
\partial_{t} f=-\nu \Lambda^{3} f-\Lambda f+\partial_{x}\left(\llbracket \mathcal{H}, f \rrbracket\left(\nu \Lambda^{3} f+\Lambda f\right)\right), \\
\left.f\right|_{t=0}=f_{0},
\end{array}\right.
$$

where $\llbracket A, B \rrbracket=A B-B A$ denotes the commutator between $A$ and $B$, the Bond number $\nu \geq 0$ represents the ratio between capillarity and gravitational effects, and the Hilbert transform $\mathcal{H}$ and the Calderón operator $\Lambda$ are defined as the following Fourier multiplier operators:

$$
\widehat{\mathcal{H f}}(n)=-i \operatorname{sgn}(n) \hat{f}(n), \quad \widehat{\Lambda f}(n)=|n| \hat{f}(n) .
$$

Equation (1.1) was derived by the authors as an asymptotic model for the Darcy flow in porous media under the assumption that the amplitude over the wavelength, a quotient known as steepness, is small [37] (see also [14, 38, 39, 41]). In the gravity driven case (when $\nu=0),(1.1)$ reads as

\footnotetext{
* Received by the editors June 5, 2019; accepted for publication (in revised form) August 6, 2020; published electronically October 14, 2020.

https://doi.org/10.1137/19M126623X

Funding: The work of the first author was supported by the Ministerio de Ciencia, Innovacion y Universidades MICIU through the project "Mathematical Analysis of Fluids and Applications," grant PID2019-109348GA-I00 and acronym "MAFyA." The work of the second author was supported by the Basque Government through the BERC 2018-2021 program and the Spanish Ministry of Economy and Competitiveness (MINECO) through BCAM Severo Ochoa excellence accreditation SEV-2017-0718 and through project MTM2017-82184-R funded by AEI/FEDER, UE, and acronym "DESFLU."

${ }^{\dagger}$ Departamento de Matemáticas, Estadística y Computación, Universidad de Cantabria, Santander, Spain (rafael.granero@unican.es).

‡Departamento de Análisis Matemático \& IMUS, Sevilla, 41012, Spain (scrobogna@us.es).
}

4937 


$$
\left\{\begin{array}{l}
\partial_{t} f+\Lambda f=\partial_{x} \llbracket \mathcal{H}, f \rrbracket \Lambda f, \\
\left.f\right|_{t=0}=f_{0} .
\end{array}\right.
$$

Although (1.1) and (1.3) seem semilinear fourth and second order nonlocal PDEs, respectively (due to terms like $f \partial_{x}^{4} f$ and $f \partial_{x}^{2} f$ ), the commutator structure of the nonlinearity implies that they are a quasilinear third and a fully nonlinear first order nonlocal PDE, respectively (see Lemma 3.1 below). This feature is key in the analysis of the Dirichlet-to-Neumann operator (cf. [3, Lemma A.1.11] and the pioneer works by Craig $[27,28,29])$.

There are several motivations to study asymptotic models of free boundary Darcy flow. One of them arises from computational reasons. The idea is, then, to simulate the asymptotic model to obtain an accurate description of the full problem at a lower computational cost. In that regard, let us briefly emphasize that the one-phase Muskat problem reveals itself as somehow harder (computationally speaking) than the twophase Muskat problem. Let us try to briefly explain the reasons for this "paradoxical" fact. In the case when there are two fluids, the gravity driven Muskat problem reads as the following single nonlocal PDE [18]:

$$
\partial_{t} f=\text { p.v. } \int_{\mathbb{R}} \frac{\left(\partial_{x} f(x)-\partial_{x} f(x-y)\right) y}{(f(x)-f(x-y))^{2}+y^{2}} d y .
$$

Simplified models for this case were provided (following heuristic ideas) by Córdoba, Gancedo, Orive [20] (see also [42]). Remarkably, when the one-phase Muskat problem is considered, the previous PDE has to be modified, and one is forced to study the following system of a nonlocal PDE and an integral equation [17]:

$$
\begin{aligned}
\partial_{t} f(x)= & \text { p.v. } \int_{\mathbb{R}} \varpi(x-y) \frac{y}{y^{2}+(f(x)-f(x-y))^{2}} d y \\
& -\partial_{x} f(x) \text { p.v. } \int_{\mathbb{R}} \varpi(x-y) \frac{f(x)-f(x-y)}{y^{2}+(f(x)-f(x-y))^{2}} d y, \\
-\partial_{x} f(x)= & \text { p.v. } \int_{\mathbb{R}} \varpi(\beta) \mathcal{B}(x, f(x), \beta, f(\beta)) d \beta \cdot\left(1, \partial_{x} f(x)\right)+\frac{\varpi(x)}{2},
\end{aligned}
$$

where $\mathcal{B}$ denotes the kernel of $\nabla^{\perp} \Delta^{-1}$, i.e.,

$$
\mathcal{B}\left(x_{1}, x_{2}, y_{1}, y_{2}\right)=\left(-\frac{x_{2}-y_{2}}{\left(x_{2}-y_{2}\right)^{2}+\left(x_{1}-y_{1}\right)^{2}}, \frac{x_{1}-y_{1}}{\left(x_{2}-y_{2}\right)^{2}+\left(x_{1}-y_{1}\right)^{2}}\right) .
$$

Thus, to write the amplitude of the vorticity in terms of the interface, one needs to invert an operator as in Córdoba, Córdoba, and Gancedo [17]. This is, mathematically and computationally, a challenging issue.

Another reason is the possibility of finding new finite time singularity scenarios. In particular, for the two-phase Muskat problem, Castro et al. [10] proved the existence of turning waves, i.e., interfaces that can be parametrized as a smooth graphs at time $t=0$ but that become smooth curves that cannot be parametrized as graphs after a finite time (see also $[7,21,22,23,33]$ ). We observe that these turning waves are interfaces such that there exist $0<T_{1}<\infty$ and

$$
\limsup _{t \rightarrow T_{1}}\left\|\partial_{x} f(t)\right\|_{L^{\infty}}=\infty .
$$

In the case of the one-phase Muskat problem, Castro et al. [11] proved the existence of curves that self-intersect in finite time in what is called a splash singularity (see 
also $[24,26,30,32]$. It remains as an interesting open problem whether the Muskat problem can have a cusp singularity, i.e., a singularity where the slope and curvature of the interface blows up while the interface remains a graph (see $[9,13,15,16,34,40]$ for global existence results). In that regard, we are optimistic enough to think that such a scenario should be simpler to prove (or discard) in an asymptotic model rather than the full problem. Taking this into consideration, the results of this paper then give conditions that excludes finite time blow ups of turning type.

1.1. Functional spaces. The space domain considered in the present article is the one-dimensional torus, i.e., $\mathbb{T}=\mathbb{R} / 2 \pi \mathbb{Z}$. The domain $\mathbb{T}$ can also be understood as the interval $[-\pi, \pi]$ endowed with periodic boundary conditions. Let $f(x)$ denote a $L^{2}$ function on $\mathbb{T}$. Then, its Fourier transform is an $\ell^{2}(\mathbb{Z})$ sequence defined as

$$
\hat{f}(n)=\hat{f}_{n}=\frac{1}{\sqrt{2 \pi}} \int_{\mathbb{T}} f(x) e^{-i x n} \mathrm{~d} x
$$

for any $n \in \mathbb{Z}$, with inverse Fourier transform

$$
f(x)=\frac{1}{\sqrt{2 \pi}} \sum_{n \in \mathbb{Z}} \hat{f}(n) e^{i x n} .
$$

Then, we define the $L^{2}$-based (homogeneous) Sobolev spaces $\dot{H}^{\alpha}(\mathbb{T})$

$$
\dot{H}^{\alpha}(\mathbb{T})=\left\{u(x) \text { such that }\|u\|_{\dot{H}^{\alpha}(\mathbb{T})}^{2}:=\sum_{k}|k|^{2 \alpha}|\hat{u}(k)|^{2}<\infty\right\} .
$$

In the present work we will need to define Sobolev spaces with fractional derivatives and integrability indexes different from two, i.e., spaces $\dot{W}^{s, p}(\mathbb{T})$ for $s \in \mathbb{R}$ and $p \in[1, \infty)$. We provide here a characterization of such spaces using using the theory of Littlewood-Paley (see Appendix A). If we consider the dyadic block $\triangle_{q}$ we can hence define the seminorm

$$
\|u\|_{\dot{W}^{s, p}(\mathbb{T})}=\left(\sum_{q \in \mathbb{Z}} 2^{p q s}\left\|\triangle_{q} u\right\|_{L^{p}(\mathbb{T})}^{p}\right)^{1 / p}
$$

It is well-known that

$$
\|u\|_{\dot{W}^{s, 2}(\mathbb{T})} \sim\|u\|_{\dot{H}^{s}(\mathbb{T})},
$$

in the sense that there exists a positive absolute constant $C>0$ such that $\frac{1}{C}\|u\|_{\dot{H}^{s}(\mathbb{T})} \leqslant$ $\|u\|_{\dot{W}^{s, 2}(\mathbb{T})} \leqslant C\|u\|_{\dot{H}^{s}(\mathbb{T})}$.

Similarly, we define the (homogeneous) Wiener spaces $\dot{A}^{\alpha}(\mathbb{T})$ and Wiener spaces with weight as

$$
\begin{aligned}
& \dot{A}^{\alpha}(\mathbb{T})=\left\{u(x) \text { such that }\|u\|_{\dot{A}^{\alpha}(\mathbb{T})}:=\sum_{k}|k|^{\alpha}|\hat{u}(k)|<\infty\right\}, \\
& \dot{A}_{\nu}^{\alpha}(\mathbb{T})=\left\{u(x) \text { such that }\|u\|_{\dot{A}_{\nu}^{\alpha}(\mathbb{T})}:=\sum_{k}|k|^{\alpha} e^{\nu|k|}|\hat{u}(k)|<\infty\right\},
\end{aligned}
$$

respectively. Obviously, $\dot{A}^{\alpha}=\dot{A}_{0}^{\alpha}$, and the functions in $A_{\nu}^{s}$ are analytic in the complex strip 


$$
\mathbb{S}_{\nu}=\{x+i y,|y|<\nu\} .
$$

We observe that (1.1) conserves the average of a solution $f$. Thus, without losing generality, we can assume this average to be zero. Thus, from this point onwards we identify the spaces $\dot{H}^{s}(\mathbb{T})$ and $H^{s}(\mathbb{T}), \dot{A}^{s}(\mathbb{T})$ and $A^{s}(\mathbb{T})$, and $\dot{A}_{\nu}^{s}(\mathbb{T})$ and $A_{\nu}^{s}(\mathbb{T})$. Then, the previously defined seminorms when restricted to average-free functions are in fact genuine norms.

Let us notice that the following embedding, valid for mean-free distributions on the one-dimensional torus

$$
\dot{H}^{1 / 2}(\mathbb{T}) \hookrightarrow L^{p}(\mathbb{T}) \forall p \in[1, \infty),
$$

and in particular the following inequality hold true for any $u \in \dot{H}^{1 / 2} \cap L^{p}$ :

$$
\|u\|_{L^{p}} \leqslant C \sqrt{p}\|u\|_{\dot{H}^{1 / 2}} .
$$

We also recall that $H^{s}(\mathbb{T})$ for $s>1 / 2$ embeds continuously in $L^{\infty}(\mathbb{T})$ with bound

$$
\|u\|_{L^{\infty}} \leqslant \frac{C}{\eta}\|u\|_{\dot{H}^{\frac{1}{2}+\eta}} .
$$

1.2. A word on scaling. We observe that, when $\nu=0,(1.1)$ is left invariant by the scaling

$$
f_{\lambda}(x, t)=\frac{1}{\lambda} f(\lambda x, \lambda t)
$$

Remarkably, this scaling is the same as in the full Muskat problem (see [25]). Then, we note that the following spaces are critical for this scaling:

$$
L^{\infty}\left(0, T ; \dot{A}^{1}(\mathbb{T})\right), L^{\infty}\left(0, T ; \dot{H}^{3 / 2}(\mathbb{T})\right), L^{\infty}\left(0, T ; \dot{W}^{1, \infty}(\mathbb{T})\right) .
$$

Let us now consider (1.1) for $\nu>0$. In this setting (1.1) reads, when expanded, as

$$
\partial_{t} f+\nu \Lambda^{3} f+\Lambda f=\nu\left[\Lambda\left(f \Lambda^{3} f\right)-\partial_{x}\left(f \partial_{x}^{3} f\right)\right]+\left[\Lambda(f \Lambda f)+\partial_{x}\left(f \partial_{x} f\right)\right] .
$$

There is no scale invariance satisfied by (1.6). Despite this fact we can rewrite (1.6) as

$$
\frac{1}{2} \partial_{t} f+\nu \Lambda^{3} f-\nu \Lambda\left(f \Lambda^{3} f\right)+\nu \partial_{x}\left(f \partial_{x}^{3} f\right)=-\frac{1}{2} \partial_{t} f-\Lambda f+\Lambda(f \Lambda f)+\partial_{x}\left(f \partial_{x} f\right)
$$

and then study separately the homogeneity of the left- and right-hand side of (1.7). Concerning the right-hand side the deductions of above still hold, and it is invariant with respect to the scaling (1.5). The left-hand side of (1.7) is invariant with respect to the scaling

$$
f_{\lambda}(x, t)=\frac{1}{\lambda} f\left(\lambda x, \lambda^{3} t\right) .
$$

We remark that the space $L_{t}^{\infty}\left(\mathbb{R}_{+}\right)$is invariant with respect to both the dilations $t \mapsto \lambda t$ and $t \mapsto \lambda^{3} t$ which characterize, respectively, (1.5) and (1.8). Then, the spaces

$$
L^{\infty}\left(0, T ; \dot{H}^{3 / 2}(\mathbb{T})\right), \quad L^{\infty}\left(0, T ; \dot{A}^{1}(\mathbb{T})\right)
$$

are, in a certain sense relatively to scale invariance, reasonable critical spaces to consider for (1.6). 


\section{Main results and discussion.}

2.1. Results for the gravity driven case (1.3). Our first result proves the existence of solution for analytic initial of arbitrary size.

TheOrem 2.1. Let $f_{0} \in A_{1}^{1}(\mathbb{T})$; there exist a short enough time $T$,

$$
0<T \leq \frac{1}{4\left\|f_{0}\right\|_{A_{1}^{1}}},
$$

and a unique mild solution to (1.3) stemming from $f_{0}$ which moreover belongs to the space

$$
f \in L^{\infty}\left(0, T ; A_{1}^{1}\right) \cap C\left([0, T], A_{0.5}^{1}\right) .
$$

This result is a sort of Cauchy-Kovalevsky theorem. However, we emphasize that the solution is less regular in time (merely $L^{\infty}$ instead of continuous) but maintains its original strip of analyticity.

Furthermore, we are able to prove a result establishing the decay of certain norms of the solution.

Proposition 2.2. Assume that the solution to (1.3) constructed in Theorem 2.1 satisfies

$$
\sup _{0 \leq t \leq T}\|f(t)\|_{\dot{A}^{1}} \leqslant 1
$$

then

$$
\begin{aligned}
& \sup _{0 \leq t \leq T} \max _{y} f(y, t) \leqslant \max _{y} f_{0}(y), \\
& \inf _{0 \leq t \leq T} \min _{y} f(y, t) \geqslant \min _{y} f_{0}(y),
\end{aligned}
$$

and, as a consequence,

$$
\sup _{0 \leq t \leq T}\|f(t)\|_{L^{\infty}} \leqslant\left\|f_{0}\right\|_{L^{\infty}}
$$

This result seems the analogue of the maximum principles known for the full Muskat problem $[5,19,23]$. In particular, when compared to the results in the previous papers, it seems that the model equation (1.3) is less stable than the full Muskat problem. The fact that the maximum principle holds if and only if $\Lambda f$ is small (see below for the proof) may seem somehow surprising. However, we should recall that the model studied in this work is only valid when the steepness parameter (i.e., a quantity akin to $\left.\|f\|_{\dot{A}^{1}}\right)$ is small. In other words, the range of validity of the PDE under consideration as a model of the full Muskat problem is related to the smallness condition guaranteeing the decay stated in the previous proposition. In this sense, since the range of validity of the model imposes restrictions in the slope of $f$, there is no contradiction between the conditional decay of the PDE under consideration and the decay with no extra assumptions of the full Muskat problem.

Once the local solution for analytic data is known, we turn our attention to the global solution for initial data satisfying certain size restrictions in critical spaces. Then, our well-posedness result for Wiener class initial data reads as follows.

Theorem 2.3. Let $f_{0} \in A^{1}$ be the initial data for (1.3). Assume that

$$
\left\|f_{0}\right\|_{\dot{A}^{1}}<1 / 2
$$

then the Cauchy problem (1.3) is globally well-posed and admits a unique solution

$$
f \in L^{\infty}\left(\mathbb{R}_{+} ; A^{1}(\mathbb{T})\right) \cap \mathcal{M}\left(\mathbb{R}_{+} ; A^{2}(\mathbb{T})\right),
$$

Copyright $@$ by SIAM. Unauthorized reproduction of this article is prohibited. 
verifying

$$
\|f(t)\|_{A^{1}} \leqslant\left\|f_{0}\right\|_{A^{1}}
$$

and

$$
\|f(t)\|_{A^{0}} \leqslant\left\|f_{0}\right\|_{A^{0}} e^{-\left(1-2\left\|f_{0}\right\|_{A^{1}}\right) t} .
$$

We observe that these are a global existence and decay for initial data in a critical space.

The next result we prove in the present manuscript is a global existence result for initial data in $H^{\frac{3}{2}} \cap H^{\frac{3}{2}+\varepsilon}, \varepsilon>0$; we suppose the initial data to be small in $H^{\frac{3}{2}}$ and of arbitrary size in $H^{\frac{3}{2}+\varepsilon}$. Very recently D. Cordoba and O. Lazar proved in [25] that it possible to construct global solutions for the two-dimensional (full) Muskat problem when the initial data is small in the space $H^{3 / 2}$ and also belongs to $H^{5 / 2}$.

The following theorem establishes a similar result for the evolution equation (1.3).

TheOrem 2.4. Let us suppose $f_{0} \in H^{\frac{3}{2}} \cap H^{\frac{3}{2}+\varepsilon}, \varepsilon>0$. There exists a constant $C>0$ independent of $\varepsilon$ and $f_{0}$ such that, if $f_{0}$ satisfies

$$
\left\|f_{0}\right\|_{H^{\frac{3}{2}}}<\min \left\{1, \frac{\varepsilon^{2}}{C\left(\left\|f_{0}\right\|_{H^{\frac{3}{2}+\varepsilon}} \log \left\|f_{0}\right\|_{H^{\frac{3}{2}+\varepsilon}}+1\right)^{2}}\right\},
$$

the Cauchy problem (1.3) is globally well-posed and admits a unique solution $f$ such that for any $T>0$

$$
\begin{aligned}
& f \in C^{0, \frac{1}{2}-\vartheta}\left([0, T) ; H^{1+\vartheta(\varepsilon+1)}\right) \cap L^{\infty}\left([0, T) ; H^{\frac{3}{2}+\varepsilon}\right) \cap L^{2}\left([0, T) ; H^{2+\varepsilon}\right), \\
& \frac{1}{2(\varepsilon+1)} \leqslant \vartheta<\frac{1}{2} .
\end{aligned}
$$

In other words, the initial data must be small in $H^{3 / 2}$ even for large $\epsilon$. Furthermore, if the initial data is large in $H^{3 / 2+\varepsilon}$, then the size condition for the initial data in $H^{3 / 2}$ is even more restrictive.

The methodology used in order to prove Theorem 2.4 differs completely from the one used in [25], since (1.3) presents a nonlinearity of polynomial type we will be able to use tools characteristic of the paradifferential calculus, we refer the reader to [6, Chapter 2]; we will hence decompose the nonlinearity in an infinite sum of elementary packets on which we will be able to highlight some nontrivial regularizing properties of (1.3).

2.2. Results for the gravity-capillary driven case (1.1). We are able to extend Theorem 2.3 to the case with surface tension.

TheOREM 2.5. Let $\nu>0$ be a fixed parameter and $f_{0} \in A^{1}$ be the initial data for (1.1). Assume that

$$
\left\|f_{0}\right\|_{\dot{A}^{1}}<1 / 2
$$

then the Cauchy problem (1.1) is globally well-posed and admits a unique solution

$$
f \in L^{\infty}\left(\mathbb{R}_{+} ; A^{1}(\mathbb{T})\right) \cap \mathcal{M}\left(\mathbb{R}_{+} ; A^{4}(\mathbb{T})\right),
$$

verifying

and

$$
\|f(t)\|_{A^{1}} \leqslant\left\|f_{0}\right\|_{A^{1}}
$$

$$
\|f(t)\|_{A^{0}} \leqslant\left\|f_{0}\right\|_{A^{0}} e^{-\left(1-2\left\|f_{0}\right\|_{A^{1}}\right)(1+\nu) t} .
$$

Copyright $@$ by SIAM. Unauthorized reproduction of this article is prohibited. 
Remark 2.6. We observe that the size condition is $\nu$-independent.

The following result is a result analogous to the one stated in Theorem 2.4 for the system (1.1) when $\nu>0$.

ThEOREM 2.7. Let us suppose that $f_{0}$ is a zero mean function on $\mathbb{T}, f_{0} \in H^{2}(\mathbb{T})$. There exists a $C>0$ such that if

$$
\left\|f_{0}\right\|_{H^{2}(\mathbb{T})} \leqslant \frac{1}{C} \min \left\{1, \nu^{-\frac{1}{4}}\right\},
$$

then there exists a unique solution $f \in \mathcal{C}\left(\mathbb{R}_{+} ; H^{2}\right) \cap L^{2}\left(\mathbb{R}_{+} ; H^{\frac{7}{2}}\right)$ of the Cauchy problem (1.1) such that for any $t>0$

$$
\|f(t)\|_{H^{2}(\mathbb{T})}^{2}+\int_{0}^{t}\left[\nu\left\|\Lambda^{3 / 2} f(s)\right\|_{H^{2}(\mathbb{T})}^{2}+\left\|\Lambda^{1 / 2} f(s)\right\|_{H^{2}(\mathbb{T})}^{2}\right] \mathrm{d} s \leqslant\left\|f_{0}\right\|_{H^{2}(\mathbb{T})}^{2} .
$$

2.3. Discussion. In this paper we prove several well-posedness results for an asymptotic model of free boundary Darcy flow. Most of them are global existence results in scale invariant spaces. In that regard, our results should be understood as nonlinear stability results rather than linear stability (even if some size conditions are imposed on the initial data). These results exclude the existence of turning wave singularities but leave open the door to cusp singularities. The occurrence of such behavior will be the object of future research.

\section{Gravity driven system (1.3).}

3.1. Proof of Theorem 2.1. The cascade of linear equations. We look for a solution of the form

$$
f(x, t)=\lambda \sum_{\ell=0}^{\infty} \lambda^{\ell} f^{(\ell)}(x, t),
$$

where $\lambda=\lambda\left(f_{0}\right)$ will be chosen below. Then, the existence of solution is reduced to the summability of a series where each term satisfies a linear problem. A similar idea can be tracked back to the works of Oseen [46] (see also [14]). Indeed, matching the appropriate terms, we find that $f^{(\ell)}$ satisfies

$$
\partial_{t} f^{(\ell)}+\Lambda f^{(\ell)}=\sum_{j=0}^{\ell-1} \Lambda\left(f^{(j)} \Lambda f^{(\ell-j-1)}\right)+\partial_{x}\left(f^{(j)} \partial_{x} f^{(\ell-j-1)}\right)
$$

with initial data

$$
f^{(\ell)}(x, 0)=0 \text { if } \ell \neq 0
$$

and

$$
f^{(0)}(x, 0)=\frac{f_{0}}{\lambda} \text { otherwise. }
$$

Then

$$
f^{(0)}=e^{-t \Lambda} \frac{f_{0}}{\lambda},
$$

and we can solve recursively for the other $f^{(\ell)}$. In particular, using that the solution of

$$
\partial_{t} u(x, t)+\Lambda u(x, t)=F(x, t), \quad u(x, t)=g(x)
$$

is given by

$$
\hat{u}(k, t)=e^{-t|k|} \hat{g}(k)+\int_{0}^{t} e^{-(t-s)|k|} \hat{F}(k, s) d s,
$$

Copyright $@$ by SIAM. Unauthorized reproduction of this article is prohibited. 
we find that

$$
\begin{aligned}
\widehat{f^{(\ell)}}(k, t)= & \sum_{j=0}^{\ell-1} \sum_{n=-\infty}^{\infty} \int_{0}^{t} e^{-(t-s)|k|}\left[|k|\left(\widehat{f^{(j)}}(n, s)|k-n| \widehat{\left.f^{(\ell-j-1}\right)}(k-n, s)\right)\right. \\
& +i k\left(\widehat{f^{(j)}}(n, s) i(k-n) f^{\widehat{(\ell-j-1)}(k-n, s))] .}\right.
\end{aligned}
$$

Thus, using

$$
|k||k-n|-k(k-n)=|k||k-n|(1-\operatorname{sgn} k \operatorname{sgn}(k-n)) \leqslant 2|k||k-n|,
$$

we have that

$$
\begin{aligned}
\partial_{x}(\widehat{\llbracket \mathcal{H}, a \rrbracket} \Lambda b) & =\sum_{n} \hat{a}(k-n) \hat{b}(n)(|k||k-n|-k(k-n)) \\
& \leqslant 2 \sum_{n}|\hat{a}(k-n) \hat{b}(n)| n \||k-n| \mid .
\end{aligned}
$$

Thus, we obtain that

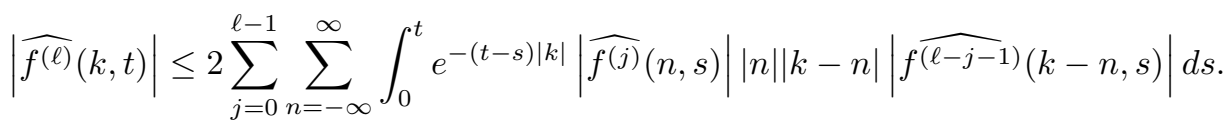

Approximate problems. We now fix $1 \ll R$ and consider the partial sum

$$
f_{R}(x, t)=\lambda \sum_{\ell=0}^{R} \lambda^{\ell} f^{(\ell)}(x, t)
$$

Then, $f_{R}$ satisfies

$$
\begin{aligned}
\partial_{t} f_{R}+\Lambda f_{R}= & \sum_{\ell=0}^{R} \lambda^{\ell+1} \sum_{j=0}^{\ell-1} \Lambda\left(f^{(j)} \Lambda f^{(\ell-j-1)}\right)+\partial_{x}\left(f^{(j)} \partial_{x} f^{(\ell-j-1)}\right) \\
= & \sum_{\ell=1}^{R} \sum_{j=0}^{\ell-1} \Lambda\left(\lambda^{j+1} f^{(j)} \lambda^{\ell-j-1+1} \Lambda f^{(\ell-j-1)}\right) \\
& +\partial_{x}\left(\lambda^{j+1} f^{(j)} \lambda^{\ell-j-1+1} \partial_{x} f^{(\ell-j-1)}\right) .
\end{aligned}
$$

Nonlinear estimates. We estimate

$$
\lambda\left\|f^{(0)}\right\|_{A_{1}^{1}} \leq\left\|f_{0}\right\|_{A_{1}^{1}} .
$$

Define

$$
\nu(\ell)=R-\ell+1 .
$$

Applying Tonelli's theorem, we find that

$$
\begin{aligned}
& \left\|f^{(\ell)}\right\|_{A_{\nu(\ell)}^{1}} \\
& \quad \leq 2 \sum_{j=0}^{\ell-1} \sum_{k=-\infty}^{\infty} \sum_{n=-\infty}^{\infty} \int_{0}^{t} e^{-(t-s)|k|}|k| e^{\nu(\ell)|k|}\left|\widehat{f^{(j)}}(n, s)\right||n||k-n|\left|f^{\widehat{(\ell-j-1})}(k-n, s)\right| d s \\
& \quad \leq 2 \sum_{j=0}^{\ell-1} \sum_{k=-\infty}^{\infty} \sum_{n=-\infty}^{\infty} \int_{0}^{t} e^{(\nu(\ell)+1)|k|}\left|\widehat{f^{(j)}}(n, s)\right||n||k-n|\left|f^{\widehat{(\ell-j-1)}}(k-n, s)\right| d s,
\end{aligned}
$$

Copyright $@$ by SIAM. Unauthorized reproduction of this article is prohibited. 
where we have used

$$
|k| \leq e^{|k|}
$$

Now we observe that, since $0 \leq j \leq \ell-1$ and $0 \leq \ell-j-1 \leq \ell-1$,

$$
\nu(\ell)+1=R-(\ell-1)+1 \leq R-j+1=\nu(j)
$$

and

$$
\nu(\ell)+1=R-(\ell-1)+1 \leq R-(\ell-j-1)+1=\nu(\ell-j-1)
$$

Thus,

$$
\begin{aligned}
& \left\|f^{(\ell)}(t)\right\|_{A_{\nu(\ell)}^{1}} \\
& \quad \leq 2 \sum_{j=0}^{\ell-1} \sum_{n, k=-\infty}^{\infty} \int_{0}^{t} e^{\nu(j)|n|} e^{\nu(\ell-j-1)|k-n|}\left|\widehat{f^{(j)}}(n, s)\right||n||k-n|\left|f^{\widehat{(\ell-j-1})}(k-n, s)\right| d s \\
& \quad \leq 2 \sum_{j=0}^{\ell-1} \int_{0}^{t}\left\|f^{(j)}(s)\right\|_{A_{\nu(j)}^{1}}\left\|f^{(\ell-j-1)}(s)\right\|_{A_{\nu(\ell-j-1)}^{1}} d s .
\end{aligned}
$$

We define the numbers

$$
\mathscr{A}_{\ell}=2\left\|f^{(\ell)}(t)\right\|_{A_{\nu(\ell)}^{1}}, \quad \mathscr{A}_{0}=1
$$

These numbers satisfy

$$
\mathscr{A}_{\ell} \leq \sum_{j=0}^{\ell-1} \int_{0}^{t} \mathscr{A}_{j} \mathscr{A}_{\ell-j-1} d s .
$$

Then, we prove by induction that

$$
\mathscr{A}_{\ell} \leq \mathscr{C}_{\ell} t^{\ell}
$$

where $\mathscr{C}_{\ell}$ are the Catalan numbers. It is known that the Catalan numbers grow like $4^{\ell}$. We also observe that

$$
\nu(\ell) \geq 1
$$

for $\ell \leq R$. As a consequence, we have the bound

$$
\begin{aligned}
\left\|f_{R}(t)\right\|_{A_{1}^{1}} & \leq \lambda\left\|f^{(0)}\right\|_{A_{1}^{1}}+\lambda \sum_{\ell=1}^{R} \lambda^{\ell}\left\|f^{(\ell)}(t)\right\|_{A_{1}^{1}} \\
& \leq \lambda\left\|f^{(0)}\right\|_{A_{1}^{1}}+\lambda \sum_{\ell=1}^{R} \lambda^{\ell}\left\|f^{(\ell)}(t)\right\|_{A_{\nu(\ell)}^{1}} \\
& \leq \lambda\left\|f^{(0)}\right\|_{A_{1}^{1}}+\frac{\lambda}{2} \sum_{\ell=1}^{R} \lambda^{\ell} \mathscr{A}_{\ell} \\
& \leq \lambda\left\|f^{(0)}\right\|_{A_{1}^{1}}+\frac{\lambda}{2} \sum_{\ell=1}^{R} \lambda^{\ell} \mathscr{C}_{\ell} t^{\ell} \\
& \leq\left\|f_{0}\right\|_{A_{1}^{1}}+\frac{\lambda}{2} \sum_{\ell=1}^{\infty}(4 \lambda t)^{\ell}
\end{aligned}
$$

Copyright (c) by SIAM. Unauthorized reproduction of this article is prohibited. 
where we have used (3.2). We choose $\lambda=\left\|f_{0}\right\|_{A_{1}^{1}}$ and note that, for $t<1 / 4\left\|f_{0}\right\|_{A_{1}^{1}}$, we obtain the bound

$$
\left\|f_{R}(t)\right\|_{A_{1}^{1}} \leq\left\|f_{0}\right\|_{A_{1}^{1}}+\frac{\left\|f_{0}\right\|_{A_{1}^{1}}}{2} \frac{4\left\|f_{0}\right\|_{A_{1}^{1}} t}{1-4\left\|f_{0}\right\|_{A_{1}^{1}} t} .
$$

Thus, we have a bound

$$
f_{R} \in L^{\infty}\left(0, T ; A_{1}^{1}\right) .
$$

Passing to the limit. This bound is independent of $R$, and then, using the Weierstrass $M$-theorem in the space $L^{\infty}\left(0, T ; A_{1}^{1}\right)$, we can pass to the limit as $R \rightarrow \infty$ and obtain

$$
\lim _{R \rightarrow \infty} f_{R}=f \in L^{\infty}\left(0, T ; A_{1}^{1}\right)
$$

Furthermore, $f_{R}$ satisfies

$$
\begin{aligned}
\widehat{f_{R}}(k, t)= & e^{-t|k|} \hat{f}_{0} \\
& +\sum_{\ell=1}^{R} \sum_{j=0}^{\ell-1} \sum_{n=-\infty}^{\infty} \int_{0}^{t} e^{-(t-s)|k|}\left(\lambda^{j+1} \widehat{f^{(j)}}(n, s) \lambda^{\ell-j-1+1} \widehat{f^{(\ell-j-1)}}(k-n, s)\right) \\
& \times[|k||k-n|-k(k-n)] .
\end{aligned}
$$

Then, using the Cauchy theorem for the product of series, the limit is a mild solution of our problem

$$
\widehat{f}(k, t)=e^{-t|k|} \hat{f}_{0}+\sum_{n=-\infty}^{\infty} \int_{0}^{t} e^{-(t-s)|k|}(\widehat{f}(n, s) \widehat{f}(k-n, s))[|k||k-n|-k(k-n)] .
$$

Time regularity. Finally, as we have that the mild solution we have just constructed is $f \in L^{\infty}\left(0, T ; A_{1}^{1}\right)$, due to the Banach scale property of the functional spaces, we also have that

$$
f, \Lambda f, \partial_{x} f \in L^{\infty}\left(0, T ; A_{0.5}^{1}\right) .
$$

Due to the fact that $f$ is a mild solution together with the previous regularity, we find that $f$ is time differentiable. Using the equation for $f$, we find that its time derivative satisfies

$$
\partial_{t} f \in L^{\infty}\left(0, T ; A_{0.5}^{1}\right) .
$$

Then, using the fundamental theorem of calculus, we can write

$$
\widehat{f}(k, t+h)-\widehat{f}(k, t)=\int_{t}^{t+h} \widehat{\partial t} f(k, s) d s,
$$

and as a consequence, we can conclude the desired regularity

$$
f \in C\left([0, T], A_{0.5}^{1}\right) .
$$

Uniqueness. We prove now that the solution with this regularity is unique. The proof is a variation of the one in [14], so we refer to it for more details. Let $f_{0} \in A_{1}^{1}$, and let us suppose there are two distinct $f_{1}$ and $f_{2}$ mild solutions of the form (3.3) stemming from $f_{0}$. Let us denote with $g=f_{1}-f_{2}$. We have that $g$ solves 


$$
\begin{aligned}
\widehat{g}(k, t)= & \sum_{n=-\infty}^{\infty} \int_{0}^{t} e^{-(t-s)|k|}\left(\widehat{g}(n, s) \widehat{f}_{1}(k-n, s)\right)[|k||k-n|-k(k-n)] \\
& +\sum_{n=-\infty}^{\infty} \int_{0}^{t} e^{-(t-s)|k|}\left(\widehat{f}_{2}(n, s) \widehat{g}_{1}(k-n, s)\right)[|k||k-n|-k(k-n)],
\end{aligned}
$$

where, by assumption, $f_{j} \in L^{\infty}\left(0, T ; A_{1}^{1}\right)$. Hence we can apply a Gronwall integral inequality and the uniform boundedness in $L^{\infty}\left(0, T ; A_{1}^{1}\right)$ to conclude that $\widehat{g}(k, t) \equiv 0$ for each $t \geqslant 0$ and $k \in \mathbb{Z}$.

3.2. Proof of Proposition 2.2. We start this section with an auxiliary lemma that establishes an integral formula for the nonlinear term.

Lemma 3.1. Let $u \in A^{1}$; the nonlinear term $\partial_{x}(\llbracket \mathcal{H}, u \rrbracket \Lambda u)$ admits the representation

$$
\begin{aligned}
\partial_{x} & (\llbracket \mathcal{H}, u \rrbracket \Lambda u) \\
& =\frac{1}{16 \pi^{2}} \text { p.v. } \int_{\mathbb{T}} \text { p.v. } \int_{\mathbb{T}} \frac{u(x)-u(x-y)}{\sin ^{2}(y / 2)} \frac{u(x-y)-u(x-y-z)}{\sin ^{2}(z / 2)} \mathrm{d} z \mathrm{~d} y+\left(\partial_{x} u(x)\right)^{2} \\
& =\frac{1}{4 \pi} \text { p.v. } \int_{\mathbb{T}} \frac{u(x)-u(x-y)}{\sin ^{2}(y / 2)} \Lambda u(x-y) \mathrm{d} y+\left(\partial_{x} u(x)\right)^{2} .
\end{aligned}
$$

Proof of Lemma 3.1. Let us assume first that $u \in C^{\infty}(\mathbb{T})$. Then we will conclude using the density of these functions in $A^{1}$. Now, we observe that for any $f, g \in A^{1}$

$$
\begin{aligned}
\llbracket \mathcal{H}, f \rrbracket g & =\frac{1}{2 \pi} \text { p.v. } \int_{\mathbb{T}} \frac{(f(x-y)-f(x)) g(x-y)}{\tan (y / 2)} \mathrm{d} y, \\
\mathcal{H}(\mathcal{H} g)(x) & =\frac{1}{2 \pi} \text { p.v. } \int_{\mathbb{T}} \frac{\mathcal{H} g(x-y)}{\tan (y / 2)} \mathrm{d} y \\
& =\frac{1}{4 \pi^{2}} \text { p.v. } \int_{\mathbb{T}} \text { p.v. } \int_{\mathbb{T}} \frac{g(x-y-z)}{\tan (z / 2)} \frac{1}{\tan (y / 2)} \mathrm{d} z \mathrm{~d} y .
\end{aligned}
$$

Thus, we obtain that, given $u \in A^{1}$

$$
\begin{aligned}
\partial_{x}(\llbracket \mathcal{H}, u \rrbracket \Lambda u) & =\frac{\partial_{x}}{2 \pi} \text { p.v. } \int_{\mathbb{T}} \frac{u(x-y)-u(x)}{\tan (y / 2)} \mathcal{H} \partial_{x} u(x-y) \mathrm{d} y \\
& =\frac{\partial_{x}}{4 \pi^{2}} \text { p.v. } \int_{\mathbb{T}} \frac{u(x-y)-u(x)}{\tan (y / 2)} \text { p.v. } \int_{\mathbb{T}} \frac{\partial_{x} u(x-y-z)}{\tan (z / 2)} \mathrm{d} z \mathrm{~d} y \\
& =\frac{\partial_{x}}{4 \pi^{2}} \text { p.v. } \int_{\mathbb{T}} \text { p.v. } \int_{\mathbb{T}} \frac{u(x-y)-u(x)}{\tan (y / 2)} \frac{\partial_{x} u(x-y-z)}{\tan (z / 2)} \mathrm{d} z \mathrm{~d} y \\
& =I_{1}+I_{2}+I_{3},
\end{aligned}
$$

where

$$
\begin{aligned}
& I_{1}=\frac{1}{4 \pi^{2}} \text { p.v. } \int_{\mathbb{T}} \text { p.v. } \int_{\mathbb{T}} \frac{\partial_{x} u(x-y)}{\tan (y / 2)} \frac{\partial_{x} u(x-y-z)}{\tan (z / 2)} \mathrm{d} z \mathrm{~d} y, \\
& I_{2}=-\frac{\partial_{x} u(x)}{4 \pi^{2}} \text { p.v. } \int_{\mathbb{T}} \text { p.v. } \int_{\mathbb{T}} \frac{1}{\tan (y / 2)} \frac{\partial_{x} u(x-y-z)}{\tan (z / 2)} \mathrm{d} z \mathrm{~d} y, \\
& I_{3}=\frac{1}{4 \pi^{2}} \text { p.v. } \int_{\mathbb{T}} \text { p.v. } \int_{\mathbb{T}} \frac{u(x-y)-u(x)}{\tan (y / 2)} \frac{\partial_{x}^{2} u(x-y-z)}{\tan (z / 2)} \mathrm{d} z \mathrm{~d} y .
\end{aligned}
$$

Copyright $@$ by SIAM. Unauthorized reproduction of this article is prohibited. 
Let us remark that $I_{3}$ is well-defined as long as $u$ has enough regularity. This term will later dissappear due to a nonlinear cancellation. We have that

$$
\begin{aligned}
I_{1}= & \frac{1}{4 \pi^{2}} \text { p.v. } \int_{\mathbb{T}} \text { p.v. } \int_{\mathbb{T}} \frac{-\partial_{y} u(x-y)}{\tan (y / 2)} \frac{\partial_{x} u(x-y-z)}{\tan (z / 2)} \mathrm{d} z \mathrm{~d} y \\
= & \frac{1}{4 \pi^{2}} \text { p.v. } \int_{\mathbb{T}} \text { p.v. } \int_{\mathbb{T}} \frac{\partial_{y}(u(x)-u(x-y))}{\tan (y / 2)} \frac{\partial_{x} u(x-y-z)}{\tan (z / 2)} \mathrm{d} z \mathrm{~d} y \\
= & \frac{1}{8 \pi^{2}} \text { p.v. } \int_{\mathbb{T}} \text { p.v. } \int_{\mathbb{T}} \frac{u(x)-u(x-y)}{\sin ^{2}(y / 2)} \frac{\partial_{x} u(x-y-z)}{\tan (z / 2)} \mathrm{d} z \mathrm{~d} y \\
& -\frac{1}{4 \pi^{2}} \text { p.v. } \int_{\mathbb{T}} \text { p.v. } \int_{\mathbb{T}} \frac{u(x)-u(x-y)}{\tan (y / 2)} \frac{\partial_{x} \partial_{y} u(x-y-z)}{\tan (z / 2)} \mathrm{d} z \mathrm{~d} y \\
= & \frac{1}{8 \pi^{2}} \text { p.v. } \int_{\mathbb{T}} \text { p.v. } \int_{\mathbb{T}} \frac{u(x)-u(x-y)}{\sin ^{2}(y / 2)} \frac{\partial_{x} u(x-y-z)}{\tan (z / 2)} \mathrm{d} z \mathrm{~d} y \\
& +\frac{1}{4 \pi^{2}} \text { p.v. } \int_{\mathbb{T}} \text { p.v. } \int_{\mathbb{T}} \frac{u(x)-u(x-y)}{\tan (y / 2)} \frac{\partial_{x}^{2} u(x-y-z)}{\tan (z / 2)} \mathrm{d} z \mathrm{~d} y \\
= & \frac{1}{8 \pi^{2}} \text { p.v. } \int_{\mathbb{T}} \text { p.v. } \int_{\mathbb{T}} \frac{u(x)-u(x-y)}{\sin ^{2}(y / 2)} \frac{-\partial_{z} u(x-y-z)}{\tan (z / 2)} \mathrm{d} z \mathrm{~d} y-I_{3} \\
= & \frac{1}{16 \pi^{2}} \text { p.v. } \int_{\mathbb{T}} \text { p.v. } \int_{\mathbb{T}} \frac{u(x)-u(x-y)}{\sin ^{2}(y / 2)} \frac{u(x-y)-u(x-y-z)}{\sin ^{2}(z / 2)} \mathrm{d} z \mathrm{~d} y-I_{3} .
\end{aligned}
$$

Using that, for zero mean functions,

$$
\mathcal{H}(\mathcal{H} g)=-g,
$$

together with (3.4), we find that

$$
I_{2}=\left(\partial_{x} u(x)\right)^{2} .
$$

Thus, collecting every term and using the density of the $C^{\infty}$ functions, we conclude the result.

Proof of Proposition 2.2. Define $X_{t}$ such that

$$
M(t)=f\left(X_{t}, t\right)=\max _{y} f(y, t) .
$$

We observe that $M(t)$ is a.e. differentiable. To see that, take two $t \leq s$, and assume that $M(t) \geq M(s)$; then

$$
\begin{aligned}
|M(t)-M(s)| & =M(t)-M(s) \\
& =f\left(X_{t}, t\right)-f\left(X_{s}, s\right) \\
& \leq f\left(X_{t}, t\right) \pm f\left(X_{t}, s\right)-f\left(X_{s}, s\right) \\
& \leqslant f\left(X_{t}, t\right)-f\left(X_{t}, s\right)
\end{aligned}
$$

where we have used

$$
f\left(X_{t}, s\right)-f\left(X_{s}, s\right) \leq 0
$$

As a consequence,

$$
|M(t)-M(s)| \leq\left\|\partial_{t} f\right\|_{L^{\infty}([0, T] \times \mathbb{T})}|t-s|,
$$

and we obtain that $M(t)$ is a Lipschitz function. Now Rademacher's theorem gives us the a.e. differentiability of $M$. Now we observe that there is a sequence of $h_{j}$ such that 


$$
\begin{aligned}
\frac{\mathrm{d}}{\mathrm{d} t} M(t) & =\lim _{h_{j} \rightarrow 0} \frac{M\left(t+h_{j}\right)-M(t)}{h_{j}} \\
& =\lim _{h_{j} \rightarrow 0} \frac{f\left(X_{t+h_{j}}, t+h_{j}\right)-f\left(X_{t}, t\right)}{h_{j}} \\
& =\lim _{h_{j} \rightarrow 0} \frac{f\left(X_{t+h_{j}}, t+h_{j}\right) \pm f\left(X_{t}, t+h_{j}\right)-f\left(X_{t}, t\right)}{h_{j}} \\
& \geq \partial_{t} f\left(X_{t}, t\right)
\end{aligned}
$$

where we have used

$$
f\left(X_{t+h_{j}}, t+h_{j}\right)-f\left(X_{t}, t+h_{j}\right) \geq 0 .
$$

Similarly, since

$$
\begin{aligned}
& f\left(X_{t-h_{j}}, t-h_{j}\right)-f\left(X_{t}, t-h_{j}\right) \geq 0, \\
\frac{\mathrm{d}}{\mathrm{d} t} M(t)= & \lim _{h_{j} \rightarrow 0} \frac{M\left(t-h_{j}\right)-M(t)}{-h_{j}} \\
= & \lim _{h_{j} \rightarrow 0} \frac{f\left(X_{t-h_{j}}, t-h_{j}\right)-f\left(X_{t}, t\right)}{-h_{j}} \\
= & \lim _{h_{j} \rightarrow 0} \frac{f\left(X_{t-h_{j}}, t-h_{j}\right) \pm f\left(X_{t}, t-h_{j}\right)-f\left(X_{t}, t\right)}{-h_{j}} \\
\leq & \partial_{t} f\left(X_{t}, t\right) .
\end{aligned}
$$

As a consequence, we find that the derivative verifies the equality

$$
\frac{\mathrm{d}}{\mathrm{d} t} M(t)=\partial_{t} f\left(X_{t}, t\right)
$$

almost everywhere in time. Using Lemma 3.1, we find that

$$
\frac{\mathrm{d}}{\mathrm{d} t} M(t)=\frac{1}{4 \pi} \int_{\mathbb{T}} \frac{M(t)-u\left(X_{t}-y\right)}{\sin ^{2}(y / 2)}\left(\Lambda u\left(X_{t}-y\right)-1\right) \mathrm{d} y .
$$

Using that

$$
|\Lambda u| \leqslant\|u(t)\|_{A^{1}},
$$

we conclude

$$
\max _{y} f(y, t) \leqslant \max _{y} f_{0}(y)
$$

If we define $x_{t}$ such that

$$
m(t)=f\left(x_{t}, t\right)=\min _{y} f(y, t),
$$

we can repeat the previous steps and conclude the result.

\subsection{Proof of Theorem 2.3.}

Step 1: A priori estimates. We start the proof providing the appropriate a priori estimates in $A^{1}$. Standard manipulations allow us to deduce the differential inequality

Copyright (c) by SIAM. Unauthorized reproduction of this article is prohibited. 


$$
\frac{\mathrm{d}}{\mathrm{d} t}\|f(t)\|_{A^{1}}+\|f(t)\|_{A^{2}} \leqslant\left\|\partial_{x}^{2}(\llbracket \mathcal{H}, f \rrbracket \Lambda f)\right\|_{A^{0}} .
$$

In Fourier variables, we have that

$$
\begin{aligned}
\partial_{x}^{2}(\widehat{\llbracket \mathcal{H}, f \rrbracket} \Lambda f) & =\partial_{x} \Lambda\left(f \Lambda \widehat{f)+\partial_{x}^{2}}\left(f \partial_{x} f\right)\right. \\
& =\hat{f}(k-m) \hat{f}(m) p(k, m)
\end{aligned}
$$

with

$$
p(k, m)=i k(|k||k-m|-k(k-m))=i k|k||k-m|\left(1-\frac{k(k-m)}{|k||k-m|}\right) .
$$

Since $\frac{k(k-m)}{|k||k-m|} \neq 1$ if and only if $m \leqslant k \leqslant 0$ and $0 \leqslant k \leqslant m$ we observe that $p \neq 0$ if and only if $0 \leqslant|k| \leqslant|m|$. We use this observation in order to argue that

$$
|p(k, m)| \leqslant 2|k|^{2}|k-m| \leq 2|m|^{2}|k-m| .
$$

Thus,

$$
\left\|\partial_{x}^{2}(\llbracket \mathcal{H}, f \rrbracket \Lambda f)\right\|_{A^{0}} \leqslant 2\|f\|_{A^{1}}\|f\|_{A^{2}} .
$$

As a consequence, we find the inequality

$$
\frac{\mathrm{d}}{\mathrm{d} t}\|f(t)\|_{A^{1}}+\left(1-2\|f(t)\|_{A^{1}}\right)\|f(t)\|_{A^{2}} \leqslant 0
$$

so that if $\left\|f_{0}\right\|_{A^{0}}<1 / 2$ a standard parabolic bootstrap argument shows that

$$
\|f(t)\|_{A^{1}}+\frac{1}{2} \int_{0}^{t}\|f(s)\|_{A^{2}} \mathrm{~d} s \leqslant\left\|f_{0}\right\|_{A^{1}} .
$$

Computations very close to the ones performed above allow us to infer that

$$
\left\|\partial_{x} \llbracket \mathcal{H}, f \rrbracket \Lambda f\right\|_{A^{0}} \leqslant 2\|f\|_{A^{1}}^{2},
$$

which we use in order to obtain the following uniform bound on the time derivative of $f$ which we will need for the compactness argument of the next step:

$$
\left\|\partial_{t} f\right\|_{A^{0}} \leqslant\|f\|_{A^{1}}+\left\|\partial_{x} \llbracket \mathcal{H}, f \rrbracket \Lambda f\right\|_{A^{0}} \leqslant\|f\|_{A^{1}}\left(1+2\|f\|_{A^{1}}\right) \leqslant\left\|f_{0}\right\|_{A^{1}}\left(1+2\left\|f_{0}\right\|_{A^{1}}\right) .
$$

Step 2: Approximated solutions and passing to the limit. To construct solutions we consider the regularized problem where the initial data is localized in Fourier space. In other words, given $f_{0}$, we consider (1.3) with the initial data $f_{0}^{N}$ where

$$
f_{0}^{N}=\sum_{n=-N}^{N} \hat{f}_{0}(n) e^{i n x} .
$$

Then, invoking Theorem 2.1, there exists a local analytic solution

$$
f^{N} \in \mathcal{C}\left(\left[0, T_{N}\right] ; A_{1 / 2}^{1}\right)
$$

for some $T_{N}>0$. Moreover the a priori estimates performed in the previous step assure us that if $\left\|f_{0}^{N}\right\|_{A^{1}} \leqslant\left\|f_{0}\right\|_{A^{1}}<1 / 2$, then $f_{N} \in L^{\infty}\left(\mathbb{R}_{+} ; A^{1}\right)$ for each $N \in \mathbb{N}$. We can hence invoke Aubin-Lions compactness lemma as it is stated in [47, Corollary 6] or [35, Lemma 5.1] in order to argue that, up to non-relabeled subsequence, $f^{N} \stackrel{N \rightarrow \infty}{\longrightarrow} f$ in $L^{q}\left([0, T] ; A^{1}\right), q \in[1, \infty)$. To prove that the limit function is a weak solution of (1.3) is standard, and the detailed computations are left to the interested reader. 
Step 3: Uniqueness. We argue by contradiction: let's assume that there exist two different solutions $f_{1}$ and $f_{2}$ starting from the same initial data $\left\|f_{0}\right\|_{A^{1}}<1 / 2$. Since $f_{0}$ satisfies the smallness condition we have that $f_{i} \in L^{\infty}\left(\mathbb{R}_{+} ; A^{1}\right) \cap L^{1}\left(\mathbb{R}_{+} ; A^{2}\right)$ and satisfy the energy inequality $(2.1)$ for $i=1,2$. Then one can prove that the difference $g=f_{1}-f_{2}$ satisfies the Cauchy problem

$$
\partial_{t} g+\Lambda g=\partial_{x}\left(\llbracket \mathcal{H}, g \rrbracket \Lambda f_{1}\right)+\partial_{x}\left(\llbracket \mathcal{H}, f_{2} \rrbracket \Lambda g\right),\left.\quad g\right|_{t=0}=0 .
$$

Performing an $A^{0}$ energy estimate on (3.6) and using the nontrivial commutation property (3.5) we deduce the energy inequality

$$
\frac{\mathrm{d}}{\mathrm{d} t}\|g\|_{A^{0}}+\|g\|_{A^{1}} \leq\|g\|_{A^{1}}\left(\left\|f_{1}\right\|_{A^{1}}+\left\|f_{2}\right\|_{A^{1}}\right) .
$$

Since $f_{i}, i=1,2$ satisfy (2.1), then using parabolic absorption and Gronwall's inequality one concludes that $g$ has to be identically nil in $A^{0}$ which implies that the solution is unique.

Step 4: Decay. We have that

$$
\frac{\mathrm{d}}{\mathrm{d} t}\|f(t)\|_{A^{0}}+\|f(t)\|_{A^{1}} \leqslant\left\|\partial_{x}(\llbracket \mathcal{H}, f \rrbracket \Lambda f)\right\|_{A^{0}} .
$$

Recalling (3.1), we find that

$$
\left\|\partial_{x}(\llbracket \mathcal{H}, f \rrbracket \Lambda f)\right\|_{A^{1}} \leqslant 2\|f\|_{A^{1}}^{2} .
$$

As a consequence, we find the inequality

$$
\frac{\mathrm{d}}{\mathrm{d} t}\|f(t)\|_{A^{0}}+\|f(t)\|_{A^{1}}\left(1-2\|f(t)\|_{A^{1}}\right) \leqslant 0 .
$$

Using the Poincaré-like inequality

$$
\|f(t)\|_{A^{0}} \leqslant\|f(t)\|_{A^{1}}
$$

we find the exponential decay

$$
\|f(t)\|_{A^{0}} \leqslant\left\|f_{0}\right\|_{A^{0}} e^{-\left(1-2\left\|f_{0}\right\|_{A^{1}}\right) t} .
$$

\subsection{Proof of Theorem 2.4.}

Step 1: A priori estimates. Equipped with the local existence of smooth solution emanating from an analytic initial data, the proof of Theorem 2.4 reduces to the derivation of appropriate energy estimates in the right Sobolev spaces. Thus, we consider $f$ to be a (space-time) smooth solution of the periodic problem (1.3).

Step 1.1: $L^{2}$ estimates. As the main nonlinear cancellation is already present at the $L^{2}(\mathbb{T})$ level, for the sake of clarity, we start with the global estimate for this low energy norm. Thus, our first goal is to prove that, if $\|f(t)\|_{H^{3 / 2}(\mathbb{T})}$ is small enough, then

$$
\|f(t)\|_{L^{2}(\mathbb{T})}^{2}+\int_{0}^{t}\left\|\Lambda^{1 / 2} f(s)\right\|_{L^{2}(\mathbb{T})}^{2} \mathrm{~d} s \leqslant\left\|f_{0}\right\|_{L^{2}(\mathbb{T})}^{2} .
$$

Let us multiply (1.3) for $f$ and integrate by parts. Then, we deduce 


$$
\begin{aligned}
\frac{1}{2} \frac{\mathrm{d}}{\mathrm{d} t}\|f(t)\|_{L^{2}(\mathbb{T})}^{2}+\left\|\Lambda^{1 / 2} f(t)\right\|_{L^{2}(\mathbb{T})}^{2} & =\int_{\mathbb{T}} f\left((\Lambda f)^{2}-\left(\partial_{x} f\right)^{2}\right) \mathrm{d} x, \\
& =\int_{\mathbb{T}} f(\mathcal{H}-1) \partial_{x} f(\mathcal{H}+1) \partial_{x} f \mathrm{~d} x, \\
& =\mathcal{N}(f, f, f),
\end{aligned}
$$

where

$$
\mathcal{N}\left(g_{1}, g_{2}, g_{3}\right)=\int_{\mathbb{T}} g_{1}(\mathcal{H}-1) \partial_{x} g_{2}(\mathcal{H}+1) \partial_{x} g_{3} \mathrm{~d} x
$$

In order to conclude the inequality (3.7), we have to prove that the following inequality holds true:

$$
|\mathcal{N}(g, h, h)| \leqslant C\|g\|_{H^{3 / 2}}\|h\|_{H^{1 / 2}}^{2} .
$$

Indeed, if we denote as $\left(\hat{g}_{n}\right)_{n \in \mathbb{Z}},\left(\hat{h}_{n}\right)_{n \in \mathbb{Z}}$ the Fourier transform of $g$ and $h$, respectively, and use the Plancherel theorem, we can rewrite $\mathcal{N}(g, h, h)$ as

$$
\begin{aligned}
\mathcal{N}(g, h, h) & =\sum_{\substack{(n, k, m) \in \mathbb{Z}^{3} \\
k+m=n}} \overline{\hat{g}_{n}}(-i \operatorname{sgn} m-1)(i m) \hat{h}_{m}(-i \operatorname{sgn} k+1)(i k) \hat{h}_{k} \\
& =\sum_{\substack{(n, k, m) \in \mathbb{Z}^{3} \\
k+m=n}} \overline{\hat{g}_{n}} m k \hat{h}_{m} \hat{h}_{k}(1+\operatorname{sgn} m \operatorname{sgn} k) .
\end{aligned}
$$

We observe that, if the addends are nonzero, $k$ and $n$ must verify

$$
0 \leqslant k \leqslant n \quad \text { or } \quad n \leqslant k \leqslant 0 .
$$

In other words,

$$
|k| \leqslant|n| .
$$

Furthermore, using the previous cancellation to find a symmetry in the series, we find that

$$
\mathcal{N}(g, h, h)=4 \sum_{\substack{(n, k) \in \mathbb{Z}^{2} \\ 0 \leqslant k \leqslant n}}(n-k) k \operatorname{Re}\left(\overline{\hat{g}}_{n} \hat{h}_{n-k} \hat{h}_{k}\right) .
$$

Thus,

$$
|\mathcal{N}(g, h, h)| \leqslant 4 \sum_{\substack{(n, k) \in \mathbb{Z}^{2} \\ 0 \leqslant k \leqslant n}}|n-k||k|\left|\hat{g}_{n}\right|\left|\hat{h}_{n-k}\right|\left|\hat{h}_{k}\right|,
$$

but since $0 \leqslant k \leqslant n$ we deduce that

$$
(n-k) k \leqslant n^{3 / 2}(n-k)^{1 / 4} k^{1 / 4},
$$

from where, using the $\ell^{2}-\ell^{2}$ Cauchy-Schwarz inequality, we can obtain the following:

$$
\begin{aligned}
& |\mathcal{N}(g, h, h)| \\
& \leqslant 4\left(\sum_{n} n^{3}\left|\hat{g}_{n}\right|^{2}\right)^{1 / 2}\left(\sum_{n}\left|\sum_{k}(n-k)^{1 / 4}\right| \hat{h}_{n-k}\left|k^{1 / 4}\right| \hat{h}_{k}||^{2}\right)^{1 / 2} .
\end{aligned}
$$

Copyright $@$ by SIAM. Unauthorized reproduction of this article is prohibited. 
Let us define the following distribution:

$$
H(x)=\mathcal{F}^{-1}\left(\left(\left|\hat{h}_{n}\right|\right)_{n}\right)(x)=\frac{1}{2 \pi} \sum_{n \in \mathbb{N}}\left|\hat{h}_{n}\right| e^{i n x} .
$$

Using the auxiliary distribution $H$ we can rewrite (3.12) as

$$
|\mathcal{N}(g, h, h)| \leqslant 4\|g\|_{H^{3 / 2}}\left\|\Lambda^{1 / 4} H \Lambda^{1 / 4} H\right\|_{L^{2}} .
$$

Using the Hölder inequality and Sobolev embeddings we deduce that

$$
\left\|\Lambda^{1 / 4} H \Lambda^{1 / 4} H\right\|_{L^{2}} \leqslant\|H\|_{\dot{W}^{\frac{1}{4}, 4}}^{2} \leqslant C\|H\|_{H^{1 / 2}}^{2},
$$

but

$$
\|H\|_{H^{1 / 2}}^{2}=\sum_{n}|n|\left|\hat{H}_{n}\right|^{2}=\sum_{n}|n|\left|\hat{h}_{n}\right|^{2}=\|h\|_{H^{1 / 2}}^{2},
$$

whence we deduce from (3.13) that

$$
|\mathcal{N}(g, h, h)| \leqslant C\|g\|_{H^{3 / 2}}\|h\|_{H^{1 / 2}}^{2} .
$$

As a consequence, we find that (3.8) now reads

$$
\frac{1}{2} \frac{\mathrm{d}}{\mathrm{d} t}\|f(t)\|_{L^{2}(\mathbb{T})}^{2}+\left\|\Lambda^{1 / 2} f(t)\right\|_{L^{2}(\mathbb{T})}^{2} \leq C\|f\|_{H^{3 / 2}}\|f\|_{H^{1 / 2}}^{2},
$$

from where we find the desired inequality (3.7).

We need now to find the appropriate bound for the higher order energy given by the $H^{3 / 2}$ norm.

Remark 3.2. It is known that the $L^{2}$ energy is a Lyapunov functional for the one-phase Muskat problem (cf. $[2,5]$ ), but for the truncated equation (1.3) such conservation is no longer valid. Let us in fact consider the analytic function

$$
\phi(x)=\frac{1}{\sqrt{2 \pi}} \sum_{n \in \mathbb{Z}} e^{-|n|} e^{i x n} ;
$$

we use the formulation (3.11) in order to argue that

$$
\begin{aligned}
\int_{\mathbb{T}} \phi\left((\Lambda \phi)^{2}-\left(\partial_{x} \phi\right)^{2}\right) \mathrm{d} x & =4 \sum_{\substack{(n, k) \in \mathbb{Z}^{2} \\
0 \leqslant k \leqslant n}}(n-k) k e^{-2 n} \\
& =4 \sum_{n \geqslant 0} e^{-2 n}\left[\frac{n^{2}(n+1)}{2}-\frac{n(n+1)(2 n+1)}{6}\right] \\
& =2 \sum_{n \geqslant 0} n e^{-2 n}\left(n^{2}-\frac{1}{3}\right) \in(0, \infty),
\end{aligned}
$$

i.e., (1.3) does not conserve the $L^{2}$ energy even for analytic functions.

Copyright $@$ by SIAM. Unauthorized reproduction of this article is prohibited. 
Step 1.2: Dyadic estimates. Let us apply the truncation operator $\triangle_{q}$ to (1.3). We then multiply the resulting equation for $\triangle_{q} f$ and integrate in space to obtain

$$
\frac{1}{2} \frac{\mathrm{d}}{\mathrm{d} t}\left\|\triangle_{q} f\right\|_{L^{2}}^{2}+\left\|\triangle_{q} \Lambda^{1 / 2} f\right\|_{L^{2}}^{2}=A_{q}-B_{q}
$$

with

$$
\begin{gathered}
A_{q}=\int \triangle_{q}(f \Lambda f) \triangle_{q} \Lambda f \mathrm{~d} x, \\
B_{q}=\int \triangle_{q}\left(f \partial_{x} f\right) \triangle_{q} \partial_{x} f \mathrm{~d} x .
\end{gathered}
$$

Using now Bony decomposition (A.2), we can split the previous terms as follows:

$$
\begin{aligned}
& A_{q}=T_{1, q}^{A}+T_{2, q}^{A}+T_{3, q}^{A}+R_{q}^{A}, \\
& B_{q}=T_{1, q}^{B}+T_{2, q}^{B}+T_{3, q}^{B}+R_{q}^{B},
\end{aligned}
$$

where

$$
\begin{aligned}
T_{1, q}^{A} & =\int S_{q-1} f\left(\triangle_{q} \Lambda f\right)^{2} \mathrm{~d} x, \\
T_{1, q}^{B} & =\int S_{q-1} f\left(\triangle_{q} \partial_{x} f\right)^{2} \mathrm{~d} x, \\
T_{2, q}^{A} & =\sum_{\left|q-q^{\prime}\right| \leqslant 4} \int\left[\triangle_{q}, S_{q^{\prime}-1} f\right] \triangle_{q^{\prime}} \Lambda f \triangle_{q} \Lambda f \mathrm{~d} x, \\
T_{2, q}^{B} & =\sum_{\left|q-q^{\prime}\right| \leqslant 4} \int\left[\triangle_{q}, S_{q^{\prime}-1} f\right] \triangle_{q^{\prime}} \partial_{x} f \triangle_{q} \partial_{x} f \mathrm{~d} x, \\
T_{3, q}^{A} & =\sum_{\left|q-q^{\prime}\right| \leqslant 4} \int\left(S_{q^{\prime}-1}-S_{q}\right) f \triangle_{q} \triangle_{q^{\prime}} \Lambda f \triangle_{q} \Lambda f \mathrm{~d} x, \\
T_{3, q}^{B} & =\sum_{\left|q-q^{\prime}\right| \leqslant 4} \int\left(S_{q^{\prime}-1}-S_{q}\right) f \triangle_{q} \triangle_{q^{\prime}} \partial_{x} f \triangle_{q} \partial_{x} f \mathrm{~d} x, \\
R_{q}^{A} & =\sum_{q^{\prime}>q-4} \int \triangle_{q}\left(\triangle_{q^{\prime}-1} f S_{q^{\prime}+2} \Lambda f\right) \triangle_{q} \Lambda f \mathrm{~d} x, \\
R_{q}^{B} & =\sum_{q^{\prime}>q-4} \int \triangle_{q}\left(\triangle_{q^{\prime}-1} f S_{q^{\prime}+2} \partial_{x} f\right) \triangle_{q} \partial_{x} f \mathrm{~d} x .
\end{aligned}
$$

We have to estimate these eight terms. The terms $T_{1}^{A}$ and $T_{1}^{B}$ are the more singular. We will make use of the commutation properties (3.10) to estimate these terms. First of all we remark that

$$
T_{1, q}^{A}-T_{1, q}^{B}=\mathcal{N}\left(S_{q-1} f, \triangle_{q} f, \triangle_{q} f\right)
$$

where the trilinear operator $\mathcal{N}$ is defined in (3.9). We can hence use inequality (3.10) and (A.3) in order to deduce

$$
\begin{aligned}
\left|T_{1, q}^{A}-T_{1, q}^{B}\right| & \leqslant C\|f\|_{H^{3 / 2}}\left\|\triangle_{q} \Lambda^{1 / 2} f\right\|_{L^{2}}^{2} \\
& \leqslant C b_{q} 2^{-2 q s}\|f\|_{H^{3 / 2}}\left\|\Lambda^{1 / 2} f\right\|_{H^{s}}^{2}
\end{aligned}
$$

for any $s \in \mathbb{R}$. 
Next we handle the remainder terms $R_{q}^{A}, R_{q}^{B}$. Indeed, we have that

$$
\begin{aligned}
R_{q}^{A} & =\sum_{q^{\prime}>q-4} \int \triangle_{q}\left(\triangle_{q^{\prime}-1} f S_{q^{\prime}+2} \Lambda f\right) \triangle_{q} \Lambda f \mathrm{~d} x \\
& \leqslant \sum_{q^{\prime}>q-4}\left\|\triangle_{q^{\prime}-1} f\right\|_{L^{\frac{2 p}{p-2}}}\left\|S_{q^{\prime}+2} \Lambda f\right\|_{L^{p}}\left\|\triangle_{q} \Lambda f\right\|_{L^{2}} .
\end{aligned}
$$

Since $H^{\frac{1}{2}}(\mathbb{T}) \hookrightarrow L^{p}(\mathbb{T})$ for any $p \in[2, \infty)$ we can say that

$$
\left\|S_{q^{\prime}+2} \Lambda f\right\|_{L^{p}} \lesssim \sqrt{p}\|f\|_{H^{3 / 2}} .
$$

Moreover due to the localization in the Fourier space and the fact that $q^{\prime}>q-4$ we deduce

$$
\begin{aligned}
\left\|\triangle_{q^{\prime}-1} f\right\|_{L^{\frac{2 p}{p-2}}}\left\|\triangle_{q} \Lambda f\right\|_{L^{2}} & \lesssim\left\|\triangle_{q^{\prime}-1} f\right\|_{L^{\frac{2 p}{p-2}}} 2^{q / 2}\left\|\triangle_{q} \Lambda^{1 / 2} f\right\|_{L^{2}} \\
& \lesssim 2^{q^{\prime} / 2}\left\|\triangle_{q^{\prime}-1} f\right\|_{L^{\frac{2 p}{p-2}}}\left\|\triangle_{q} \Lambda^{1 / 2} f\right\|_{L^{2}} \\
& \lesssim\left\|\triangle_{q^{\prime}-1} \Lambda^{1 / 2} f\right\|_{L^{\frac{2 p}{p-2}}}\left\|\triangle_{q} \Lambda^{1 / 2} f\right\|_{L^{2}} .
\end{aligned}
$$

We use (A.3), (3.18), and a summation in $q^{\prime}$ in order to transform (3.17) into

$$
R_{q}^{A} \lesssim b_{q} 2^{-2 q s} \sqrt{p}\|f\|_{H^{3 / 2}}\left\|\Lambda^{1 / 2} f\right\|_{H^{s}}\left\|\Lambda^{1 / 2} f\right\|_{\dot{W}^{s, \frac{2 p}{p-2}}} .
$$

Using the Sobolev inequality (1.4), we can also obtain the following estimate for the term $R_{q}^{A}$ :

$$
\begin{aligned}
R_{q}^{A} & =\sum_{q^{\prime}>q-4}\left\|S_{q^{\prime}+2} \Lambda f\right\|_{L^{\infty}}\left\|\triangle_{q^{\prime}-1} \Lambda^{1 / 2} f\right\|_{L^{2}}\left\|\triangle_{q} \Lambda^{1 / 2} f\right\|_{L^{2}} \\
& \lesssim b_{q} 2^{-2 q s} \frac{1}{\delta}\|f\|_{H^{\frac{3}{2}+\delta}}\left\|\Lambda^{1 / 2} f\right\|_{H^{s}}^{2}
\end{aligned}
$$

for any $0<\delta \ll 1$.

A very similar procedure allows us to deduce the following bound:

$$
\begin{aligned}
& R_{q}^{B} \lesssim b_{q} 2^{-2 q s} \sqrt{p}\|f\|_{H^{3 / 2}}\left\|\Lambda^{1 / 2} f\right\|_{H^{s}}\left\|\Lambda^{1 / 2} f\right\|_{\dot{W}^{s, \frac{2 p}{p-2}}}, \\
& R_{q}^{B} \lesssim b_{q} 2^{-2 q s} \frac{1}{\delta}\|f\|_{H^{\frac{3}{2}+\delta}}\left\|\Lambda^{1 / 2} f\right\|_{H^{s}}^{2} .
\end{aligned}
$$

We turn now our attention to the element $T_{2}^{A}$. Using Lemma A.1 we obtain

$$
\begin{aligned}
\left|T_{2, q}^{A}\right| & \leq \sum_{\left|q-q^{\prime}\right| \leqslant 4}\left\|\left[\triangle_{q}, S_{q^{\prime}-1} f\right] \triangle_{q^{\prime}} \Lambda f\right\|_{L^{2}}\left\|\triangle_{q} \Lambda f\right\|_{L^{2}} \\
& \leq \sum_{\left|q-q^{\prime}\right| \leqslant 4} 2^{-q}\left\|\triangle_{q} \Lambda f\right\|_{L^{2}}\|\Lambda f\|_{L^{p}}\left\|\triangle_{q^{\prime}} \Lambda f\right\|_{L^{\frac{2 p}{p-2}}} \\
& \lesssim \sum_{\left|q-q^{\prime}\right| \leqslant 4}\left\|\triangle_{q} \Lambda^{1 / 2} f\right\|_{L^{2}}\|\Lambda f\|_{L^{p}}\left\|\triangle_{q^{\prime}} \Lambda^{1 / 2} f\right\|_{L^{\frac{2 p}{p-2}}} \\
& \lesssim b_{q} 2^{-2 q s} \sqrt{p}\|f\|_{H^{3 / 2}}\left\|\Lambda^{1 / 2} f\right\|_{H^{s}}\left\|\Lambda^{1 / 2} f\right\|_{\dot{W}^{s, \frac{2 p}{p-2}}} .
\end{aligned}
$$

As a consequence, we can conclude the following bound:

Copyright (c) by SIAM. Unauthorized reproduction of this article is prohibited. 


$$
\left|T_{2, q}^{A}\right| \lesssim b_{q} 2^{-2 q s} \frac{1}{\delta}\|f\|_{H^{\frac{3}{2}+\delta}}\left\|\Lambda^{1 / 2} f\right\|_{H^{s}}^{2}
$$

Similarly, we can deduce the bounds

$$
\begin{aligned}
& \left|T_{2, q}^{B}\right| \lesssim b_{q} 2^{-2 q s} \sqrt{p}\|f\|_{H^{3 / 2}}\left\|\Lambda^{1 / 2} f\right\|_{H^{s}}\left\|\Lambda^{1 / 2} f\right\|_{\dot{W}^{s, \frac{2 p}{p-2}}}, \\
& \left|T_{2, q}^{B}\right| \lesssim b_{q} 2^{-2 q s} \frac{1}{\delta}\|f\|_{H^{\frac{3}{2}+\delta}}\left\|\Lambda^{1 / 2} f\right\|_{H^{s}}^{2} .
\end{aligned}
$$

The estimates

$$
\left|T_{3, q}^{A}\right|+\left|T_{3, q}^{B}\right| \lesssim b_{q} 2^{-2 q s}\|f\|_{H^{3 / 2}}\left\|\Lambda^{1 / 2} f\right\|_{H^{s}}^{2}
$$

can be easily obtained since the terms composing the elements $T_{3, q}^{A}, T_{3, q}^{B}$ are all localized in dyadic annuli, and hence these terms are more regular than the ones estimated above. We note that

$$
\begin{aligned}
\frac{1}{2} \frac{\mathrm{d}}{\mathrm{d} t}\left\|\triangle_{q} f\right\|_{L^{2}}^{2}+\left\|\triangle_{q} \Lambda^{1 / 2} f\right\|_{L^{2}}^{2} \leqslant & C b_{q} 2^{-2 q s}\left[\|f\|_{H^{3 / 2}}\left\|\Lambda^{1 / 2} f\right\|_{H^{s}}^{2}\right. \\
& \left.+\sqrt{p}\|f\|_{H^{3 / 2}}\left\|\Lambda^{1 / 2} f\right\|_{H^{s}}\left\|\Lambda^{1 / 2} f\right\|_{\dot{W}^{s, \frac{2 p}{p-2}}}\right]
\end{aligned}
$$

where we have used (3.16), (3.19), (3.21), (3.23), (3.25), and (3.27). Now, multiplying the above equation for $2^{2 q s}$ and summing in $q \in \mathbb{Z}$, we obtain

$$
\begin{aligned}
\frac{1}{2} \frac{\mathrm{d}}{\mathrm{d} t}\|f\|_{H^{s}}^{2}+\left\|\Lambda^{1 / 2} f\right\|_{H^{s}}^{2} \leqslant C & {\left[\|f\|_{H^{3 / 2}}\left\|\Lambda^{1 / 2} f\right\|_{H^{s}}^{2}\right.} \\
& \left.+\sqrt{p}\|f\|_{H^{3 / 2}}\left\|\Lambda^{1 / 2} f\right\|_{H^{s}}\left\|\Lambda^{1 / 2} f\right\|_{\dot{W}^{s, \frac{2 p}{p-2}}}\right]
\end{aligned}
$$

Using (3.16), (3.20), (3.22), (3.24), (3.26), and (3.27) instead we deduce

$$
\frac{1}{2} \frac{\mathrm{d}}{\mathrm{d} t}\|f\|_{H^{s}}^{2}+\left\|\Lambda^{1 / 2} f\right\|_{H^{s}}^{2} \leqslant C\left[\|f\|_{H^{3 / 2}}+\frac{1}{\delta}\|f\|_{H^{\frac{3}{2}+\delta}}\right]\left\|\Lambda^{1 / 2} f\right\|_{H^{s}}^{2} .
$$

Step 1.3: $\boldsymbol{H}^{\mathbf{3} / 2}$ estimates. We can now simply consider the estimate (3.28) in which we set $s=3 / 2$ and

$$
p=p_{\varepsilon}=\frac{1}{\varepsilon}
$$

in order to deduce

$$
\begin{aligned}
\frac{1}{2} \frac{\mathrm{d}}{\mathrm{d} t}\|f\|_{H^{3 / 2}}^{2}+\left\|\Lambda^{1 / 2} f\right\|_{H^{3 / 2}}^{2} \leqslant C & \|f\|_{H^{3 / 2}}\left\|\Lambda^{1 / 2} f\right\|_{H^{3 / 2}}^{2} \\
& \left.+\frac{1}{\sqrt{\varepsilon}}\|f\|_{H^{3 / 2}}\left\|\Lambda^{1 / 2} f\right\|_{H^{3 / 2}}\left\|\Lambda^{1 / 2} f\right\|_{\dot{W}^{\frac{3}{2}}, \frac{2 p}{p^{-2}}}\right) .
\end{aligned}
$$

Next we remark that $H^{\frac{3}{2}+\varepsilon} \hookrightarrow \dot{W}^{\frac{3}{2}, \frac{2 p}{p-2}}$, whence 


$$
\begin{aligned}
\frac{1}{2} \frac{\mathrm{d}}{\mathrm{d} t}\|f\|_{H^{3 / 2}}^{2}+\left\|\Lambda^{1 / 2} f\right\|_{H^{3 / 2}}^{2} \leqslant & C\left(\|f\|_{H^{3 / 2}}\left\|\Lambda^{1 / 2} f\right\|_{H^{3 / 2}}^{2}\right. \\
& \left.+\frac{1}{\sqrt{\varepsilon}}\|f\|_{H^{3 / 2}}\left\|\Lambda^{1 / 2} f\right\|_{H^{3 / 2}}\left\|\Lambda^{1 / 2} f\right\|_{H^{\frac{3}{2}+\varepsilon}}\right)
\end{aligned}
$$

Step 1.4: $\boldsymbol{H}^{\frac{3}{2}+\varepsilon}$ estimates. We invoke the estimate (3.29) with $s=\frac{3}{2}+\varepsilon$ and $0<\delta \ll \varepsilon$ :

$$
\frac{1}{2} \frac{\mathrm{d}}{\mathrm{d} t}\|f\|_{H^{\frac{3}{2}+\varepsilon}}^{2}+\left\|\Lambda^{1 / 2} f\right\|_{H^{\frac{3}{2}+\varepsilon}}^{2} \leqslant C\left(\|f\|_{H^{3 / 2}}+\frac{1}{\delta}\|f\|_{H^{\frac{3}{2}+\delta}}\right)\left\|\Lambda^{1 / 2} f\right\|_{H^{\frac{3}{2}+\varepsilon}}^{2} .
$$

Using interpolation between Sobolev spaces we deduce

$$
\|f\|_{H^{\frac{3}{2}+\delta}} \leqslant\|f\|_{H^{3 / 2}}^{1-\frac{\delta}{\varepsilon}}\|f\|_{H^{\frac{3}{2}+\varepsilon}}^{\frac{\delta}{\varepsilon}}
$$

from where we obtain that

$$
\frac{1}{2} \frac{\mathrm{d}}{\mathrm{d} t}\|f\|_{H^{\frac{3}{2}+\varepsilon}}^{2}+\left\|\Lambda^{1 / 2} f\right\|_{H^{\frac{3}{2}+\varepsilon}}^{2} \leqslant C\left(\|f\|_{H^{3 / 2}}+\frac{1}{\delta}\|f\|_{H^{3 / 2}}^{1-\frac{\delta}{\varepsilon}}\|f\|_{H^{\frac{3}{2}+\varepsilon}}^{\frac{\delta}{\varepsilon}}\right)\left\|\Lambda^{1 / 2} f\right\|_{H^{\frac{3}{2}+\varepsilon}}^{2} .
$$

Step 1.5: Closing the estimates. In this section we conclude the high order energy estimates. We sum up (3.30) and (3.31) to find that

$$
\begin{aligned}
\frac{1}{2} \frac{\mathrm{d}}{\mathrm{d} t} & \left(\|f\|_{H^{3 / 2}}^{2}+\|f\|_{H^{\frac{3}{2}+\varepsilon}}^{2}\right)+\left(\left\|\Lambda^{1 / 2} f\right\|_{H^{3 / 2}}^{2}+\left\|\Lambda^{1 / 2} f\right\|_{H^{\frac{3}{2}+\varepsilon}}^{2}\right) \\
\leqslant & C\left(1+\frac{1}{\sqrt{\varepsilon}}\right)\|f\|_{H^{3 / 2}}\left\|\Lambda^{1 / 2} f\right\|_{H^{3 / 2}}^{2} \\
& +C\left(\left(1+\frac{1}{\sqrt{\varepsilon}}\right)\|f\|_{H^{3 / 2}}+\frac{1}{\delta}\|f\|_{H^{3 / 2}}^{1-\frac{\delta}{\varepsilon}}\|f\|_{H^{\frac{3}{2}+\varepsilon}}^{\frac{\delta}{2}}\right)\left\|\Lambda^{1 / 2} f\right\|_{H^{\frac{3}{2}+\varepsilon}}^{2}
\end{aligned}
$$

We define

$$
\beta=\beta\left(f_{0}\right)=\frac{\left\|f_{0}\right\|_{H^{\frac{3}{2}+\varepsilon}}}{\left\|f_{0}\right\|_{H^{\frac{3}{2}}}}>1 .
$$

We want now to absorb the contribution of

$$
\frac{1}{\delta}\|f\|_{H^{3 / 2}}^{1-\frac{\delta}{\varepsilon}}\|f\|_{H^{\frac{3}{2}+\varepsilon}}^{\frac{\delta}{\varepsilon}}\left\|\Lambda^{1 / 2} f\right\|_{H^{\frac{3}{2}+\varepsilon}}^{2}
$$

appearing in the right-hand side of (3.32) in the parabolic term in the left-hand side of the same estimate. In order to do so we must split the proof according to the explicit value of $\beta$.

Case 1: $\boldsymbol{\beta} \leqslant \mathbf{2}$. In this setting we fix $\delta=\varepsilon / 2$; hence $\beta^{\delta / \varepsilon} / \delta \leqslant 2^{3 / 2} / \varepsilon$. Thus

$$
\frac{1}{\delta}\|f\|_{H^{3 / 2}}^{1-\frac{\delta}{\varepsilon}}\|f\|_{H^{\frac{3}{2}+\varepsilon}}^{\frac{\delta}{\varepsilon}}=\frac{1}{\delta}\|f\|_{H^{\frac{3}{2}}} \beta^{\delta / \varepsilon} \leqslant \frac{2^{3 / 2}}{\varepsilon}\|f\|_{H^{\frac{3}{2}}} .
$$

So, if the initial data is small enough in $H^{3 / 2}$, then (3.33) is as small as needed in order to absorb the desired term.

Copyright $@$ by SIAM. Unauthorized reproduction of this article is prohibited. 
Case 2: $\boldsymbol{\beta}>\mathbf{2}$. In this setting we can define

$$
\delta=\delta\left(f_{0}\right)=\varepsilon \log _{\beta} 2<\varepsilon .
$$

The value of $\delta$ has been defined so that

$$
\beta^{\delta / \varepsilon}=2 .
$$

We compute

$$
\frac{1}{\delta}\left\|f_{0}\right\|_{H^{3 / 2}}^{1-\frac{\delta}{\varepsilon}}\left\|f_{0}\right\|_{H^{\frac{3}{2}+\varepsilon}}^{\frac{\delta}{\varepsilon}}=\frac{1}{\delta}\left\|f_{0}\right\|_{H^{\frac{3}{2}}} \beta^{\delta / \varepsilon}=\frac{2}{\varepsilon \log _{\beta} 2}\left\|f_{0}\right\|_{H^{\frac{3}{2}}}
$$

We want to make sure that

$$
\frac{2 C}{\varepsilon \log _{\beta} 2}\left\|f_{0}\right\|_{H^{\frac{3}{2}}}<1 / 2
$$

Thus, simplifying the previous expression, we obtain that our goal is to prove that the following inequality holds:

$$
\left\|f_{0}\right\|_{H^{\frac{3}{2}}} \log \left(\frac{\left\|f_{0}\right\|_{H^{\frac{3}{2}+\varepsilon}}}{\left\|f_{0}\right\|_{H^{\frac{3}{2}}}}\right)=\left\|f_{0}\right\|_{H^{\frac{3}{2}}}\left(\log \left\|f_{0}\right\|_{H^{\frac{3}{2}+\varepsilon}}-\log \left\|f_{0}\right\|_{H^{\frac{3}{2}}}\right)<\frac{\varepsilon \log 2}{4 C} .
$$

We will now use the algebraic inequality $-x \log x \leq \sqrt{x}, x \in[0,1]$ to obtain

$$
\begin{aligned}
\left\|f_{0}\right\|_{H^{\frac{3}{2}}} \log \left(\frac{\left\|f_{0}\right\|_{H^{\frac{3}{2}+\varepsilon}}}{\left\|f_{0}\right\|_{H^{\frac{3}{2}}}}\right) & =\left\|f_{0}\right\|_{H^{\frac{3}{2}}}\left(\log \left\|f_{0}\right\|_{H^{\frac{3}{2}+\varepsilon}}-\log \left\|f_{0}\right\|_{H^{\frac{3}{2}}}\right) \\
& \leq\left\|f_{0}\right\|_{H^{\frac{3}{2}}}^{1 / 2}\left(\left\|f_{0}\right\|_{H^{\frac{3}{2}+\varepsilon}}^{1 / 2} \log \left\|f_{0}\right\|_{H^{\frac{3}{2}+\varepsilon}}+1\right) .
\end{aligned}
$$

It is now clear that, under the assumptions of the statement, we have that

$$
\left\|f_{0}\right\|_{H^{\frac{3}{2}}}^{1 / 2}\left(\left\|f_{0}\right\|_{H^{\frac{3}{2}+\varepsilon}}^{1 / 2} \log \left\|f_{0}\right\|_{H^{\frac{3}{2}+\varepsilon}}+1\right)<\frac{\varepsilon \log 2}{4 C} .
$$

In other words, we can now collect the estimates (3.33) and (3.34) to obtain that if the initial data satisfies the condition

$$
\left\|f_{0}\right\|_{H^{\frac{3}{2}}}<\min \left\{1, \frac{\varepsilon^{2} \log ^{2} 2}{16 C^{2}\left(\left\|f_{0}\right\|_{H^{\frac{3}{2}+\varepsilon}} \log \left\|f_{0}\right\|_{H^{\frac{3}{2}+\varepsilon}}+1\right)^{2}}\right\},
$$

then

$$
\frac{1}{\delta}\|f\|_{H^{3 / 2}}^{1-\frac{\delta}{\varepsilon}}\|f\|_{H^{\frac{3}{2}+\varepsilon}}^{\frac{\delta}{\varepsilon}}\left\|\Lambda^{1 / 2} f\right\|_{H^{\frac{3}{2}+\varepsilon}}^{2}<\frac{1}{2}\left\|\Lambda^{1 / 2} f\right\|_{H^{\frac{3}{2}+\varepsilon}}^{2} .
$$

Thus, due to the previous energy estimates, we can absorb the contribution from the nonlinear terms and conclude that

$$
\begin{aligned}
& \|f(t)\|_{H^{3 / 2}}^{2}+\|f(t)\|_{H^{\frac{3}{2}+\varepsilon}}^{2}+\int_{0}^{t}\left(\left\|\Lambda^{1 / 2} f(s)\right\|_{H^{3 / 2}}^{2}+\left\|\Lambda^{1 / 2} f(s)\right\|_{H^{\frac{3}{2}+\varepsilon}}^{2}\right) \mathrm{d} s \\
& \quad \leqslant\left\|f_{0}\right\|_{H^{3 / 2}}^{2}+\left\|f_{0}\right\|_{H^{\frac{3}{2}+\varepsilon}}^{2} .
\end{aligned}
$$

The above equation implies, hence, that smooth solutions stemming from small $H^{3 / 2}$ initial data are nonlinearly stable in $H^{\frac{3}{2}} \cap H^{\frac{3}{2}+\varepsilon}$.

Copyright $@$ by SIAM. Unauthorized reproduction of this article is prohibited. 
Step 1.6: Estimates for the time derivative. The purpose of this section is to find estimates for the time derivative of the solution. In particular, we want to obtain the following:

$$
\begin{aligned}
& \int_{0}^{t}\left\|\partial_{t} f(s)\right\|_{H^{1}}^{2} d s \\
& \leqslant C\left\{\left(\left\|f_{0}\right\|_{H^{3 / 2}}^{2}+\left\|f_{0}\right\|_{H^{\frac{3}{2}+\varepsilon}}^{2}\right)\left[1+\left(\left\|f_{0}\right\|_{H^{3 / 2}}^{2}+\left\|f_{0}\right\|_{H^{\frac{3}{2}+\varepsilon}}^{2}\right)\left(1+\frac{1}{\sqrt{\varepsilon}}\right)\right]\right. \\
& \left.\quad+\left\|f_{0}\right\|_{H^{3 / 2}}^{3}+\left\|f_{0}\right\|_{H^{\frac{3}{2}+\varepsilon}}^{3}\right\} .
\end{aligned}
$$

We multiply (1.3) by $\partial_{t} \Lambda^{2} f$ and integrate in $\mathbb{T} \times(0, T)$ to obtain

$$
\left\|\partial_{t} \Lambda f\right\|_{L_{T}^{2} L^{2}}^{2}+\left(\|f(t)\|_{H^{\frac{3}{2}}}^{2}-\left\|f_{0}\right\|_{H^{\frac{3}{2}}}^{2}\right)=I_{1}+I_{2},
$$

where

$$
I_{1}=\int_{0}^{T} \int_{\mathbb{T}} \Lambda(f \Lambda f) \partial_{t} \Lambda^{2} f \mathrm{~d} x \mathrm{~d} s
$$

and

$$
I_{2}=\int_{0}^{T} \int_{\mathbb{T}} \partial_{x}\left(f \partial_{x} f\right) \partial_{t} \Lambda^{2} f \mathrm{~d} x \mathrm{~d} s
$$

Using the identity $\Lambda^{2}=-\partial_{x}^{2}$ and integrating by parts we can deduce the following equalities:

$$
\begin{aligned}
I_{1} & =\int_{0}^{T} \int_{\mathbb{T}} \partial_{x} f \Lambda f \partial_{t} \Lambda \partial_{x} f \mathrm{~d} x \mathrm{~d} s+\int_{0}^{T} \int_{\mathbb{T}} f \Lambda \partial_{x} f \partial_{t} \Lambda \partial_{x} f \mathrm{~d} x \mathrm{~d} s \\
& =I_{1,1}+I_{1,2}, \\
I_{2} & =-\int_{0}^{T} \int_{\mathbb{T}}\left(\partial_{x} f\right)^{2} \partial_{t} \partial_{x}^{2} f \mathrm{~d} x \mathrm{~d} s-\int_{0}^{T} \int_{\mathbb{T}} f \partial_{x}^{2} f \partial_{t} \partial_{x}^{2} f \mathrm{~d} x \mathrm{~d} s \\
& =I_{2,1}+I_{2,2} .
\end{aligned}
$$

We hence obtain that

$$
\begin{aligned}
I_{1,2}+I_{2,2} & =\frac{1}{2} \int_{0}^{T} \int_{\mathbb{T}} f \partial_{t}\left[\left(\Lambda \partial_{x} f\right)^{2}-\left(\partial_{x}^{2} f\right)^{2}\right] \mathrm{d} x \mathrm{~d} s \\
& =\left.\frac{1}{2} \mathcal{N}\left(f, \partial_{x} f, \partial_{x} f\right)\right|_{0} ^{T}-\frac{1}{2} \int_{0}^{T} \mathcal{N}\left(\partial_{t} f, \partial_{x} f, \partial_{x} f\right) \mathrm{d} s \\
& =J_{1}+J_{2} .
\end{aligned}
$$

The term $J_{1}$ can be estimated using (3.10), and we deduce

$$
J_{1} \lesssim\|f(T)\|_{H^{3 / 2}}^{3}+\left\|f_{0}\right\|_{H^{3 / 2}}^{3}
$$

The term $J_{2}$ can be estimated in a similar way. In particular, we obtain for any $\epsilon>0$

Copyright $@$ by SIAM. Unauthorized reproduction of this article is prohibited. 


$$
\begin{aligned}
J_{2} & \lesssim \int_{0}^{T}\left\|\partial_{t} f\right\|_{H^{1}}\left\|\Lambda^{3 / 2} f\right\|_{L^{4}}^{2} \mathrm{~d} s \\
& \lesssim \int_{0}^{T}\left\|\partial_{t} f\right\|_{H^{1}}\|f\|_{H^{3 / 2}}\left\|\Lambda^{1 / 2} f\right\|_{H^{3 / 2}} \mathrm{~d} s \\
& \leqslant \epsilon\left\|\partial_{t} f\right\|_{L_{T}^{2} H^{1}}^{2}+\frac{C}{\epsilon}\|f\|_{L_{T}^{\infty} H^{3 / 2}}^{2}\left\|\Lambda^{1 / 2} f\right\|_{L_{T}^{2} H^{3 / 2}}^{2}
\end{aligned}
$$

We have to estimate the term $I_{1,1}+I_{2,1}$. We find that

$$
\begin{aligned}
I_{1,1} & =\int_{0}^{T} \int_{\mathbb{T}} \partial_{x} f \Lambda f \partial_{t} \Lambda \partial_{x} f \mathrm{~d} x \mathrm{~d} s \\
& \sim \int_{0}^{T} \int_{\mathbb{T}} \Lambda^{2} f \Lambda f \partial_{t} \Lambda f \mathrm{~d} x \mathrm{~d} s \\
& \lesssim\left\|\Lambda^{2} f\right\|_{L_{T}^{2} L^{\frac{2 p_{\varepsilon}}{p_{\varepsilon}-2}}}\|\Lambda f\|_{L_{T}^{\infty} L^{p_{\varepsilon}}}\left\|\partial_{t} \Lambda f\right\|_{L_{T}^{2} L^{2}} \\
& \lesssim \frac{1}{\sqrt{\varepsilon}}\left\|\Lambda^{1 / 2} f\right\|_{L_{T}^{2} H^{\frac{3}{2}+\varepsilon}}\|f\|_{L_{T}^{\infty} H^{3 / 2}}\left\|\partial_{t} f\right\|_{L_{T}^{2} H^{1}} \\
& \leqslant \eta\left\|\partial_{t} f\right\|_{L_{T}^{2} H^{1}}^{2}+\frac{C}{\eta \sqrt{\varepsilon}}\left\|\Lambda^{1 / 2} f\right\|_{L_{T}^{2} H^{\frac{3}{2}+\varepsilon}}^{2}\|f\|_{L_{T}^{\infty} H^{3 / 2}}^{2}
\end{aligned}
$$

where $\eta>0$ is arbitrary. The term $I_{2,1}$ is completely analogous to $I_{1,1}$ and can be handled in a similar way, and we obtain the estimate

$$
I_{2,1} \leqslant \eta\left\|\partial_{t} f\right\|_{L_{T}^{2} H^{1}}^{2}+\frac{C}{\eta \sqrt{\varepsilon}}\left\|\Lambda^{1 / 2} f\right\|_{L_{T}^{2} H^{\frac{3}{2}+\varepsilon}}^{2}\|f\|_{L_{T}^{\infty} H^{3 / 2}}^{2} .
$$

Using the estimates $(3.38),(3.39),(3.40)$ in (3.37) we obtain that

$$
\begin{aligned}
(1-2 \eta)\left\|\partial_{t} f\right\|_{L_{T}^{2} H^{1}}^{2} & \leqslant C\left[\left\|f_{0}\right\|_{H^{\frac{3}{2}}}^{2}-\|f(T)\|_{H^{\frac{3}{2}}}^{2}+\|f(T)\|_{H^{3 / 2}}^{3}+\left\|f_{0}\right\|_{H^{3 / 2}}^{3}\right. \\
& \left.+\frac{1}{\eta}\left(\left\|\Lambda^{1 / 2} f\right\|_{L_{T}^{2} H^{3 / 2}}^{2}+\frac{1}{\sqrt{\varepsilon}}\left\|\Lambda^{1 / 2} f\right\|_{L_{T}^{2} H^{\frac{3}{2}+\varepsilon}}^{2}\right)\|f\|_{L_{T}^{\infty} H^{3 / 2}}^{2}\right] .
\end{aligned}
$$

We fix $\eta=1 / 4$ and use (3.35) in order to deduce the bound

$$
\begin{aligned}
& \frac{\left\|\partial_{t} f\right\|_{L_{T}^{2} H^{1}}^{2}}{2} \\
& \qquad C\left\{\left(\left\|f_{0}\right\|_{H^{3 / 2}}^{2}+\left\|f_{0}\right\|_{H^{\frac{3}{2}+\varepsilon}}^{2}\right)\left[1+\left(\left\|f_{0}\right\|_{H^{3 / 2}}^{2}+\left\|f_{0}\right\|_{H^{\frac{3}{2}+\varepsilon}}^{2}\right)\left(1+\frac{1}{\sqrt{\varepsilon}}\right)\right]\right. \\
& \left.\quad+\left\|f_{0}\right\|_{H^{3 / 2}}^{3}+\left\|f_{0}\right\|_{H^{\frac{3}{2}+\varepsilon}}^{3}\right\} .
\end{aligned}
$$

Step 2: Approximated solutions and passing to the limit. The a priori estimates provided before the proof of Theorem 2.4 become a standard approximation argument which we outline here. Let us define the truncation operator

$$
\mathcal{J}_{n} u(x)=\frac{1}{\sqrt{2 \pi}} \sum_{|k| \leqslant n} \hat{u}_{k} e^{i k \cdot x}=\mathcal{F}^{-1}\left(\left(1_{\{|k| \leqslant n\}}(k) \hat{u}_{k}\right)_{k}\right) .
$$

Copyright $@$ by SIAM. Unauthorized reproduction of this article is prohibited. 
We apply this truncation operator to the initial data $f_{0}$. Then, this truncated initial data is analytic, and, invoking Theorem 2.1, we obtain the existence of smooth (in time and space) local solution approximate solutions. We can use the previous estimates (3.35) to deduce that the local approximate solutions are actually globally defined as $H^{3 / 2+\varepsilon}$ functions. The sequence of approximate solutions $\left(f_{n}\right)_{n}$ is bounded uniformly in $n$ and $T$ in the spaces

$$
L^{\infty}\left([0, T) ; H^{\frac{3}{2}} \cap H^{\frac{3}{2}+\varepsilon}\right) \cap L^{2}\left([0, T) ; H^{2} \cap H^{2+\varepsilon}\right),
$$

so there exists a weak limit

$$
f \in L^{\infty}\left([0, T) ; H^{\frac{3}{2}+\varepsilon}\right) \cap L^{2}\left(0, T ; H^{2+\varepsilon}\right) .
$$

Furthermore, estimate (3.36) ensures that the sequence of approximate solutions $\left(f_{n}\right)_{n}$ is uniformly bounded in

$$
H^{1}\left([0, T) ; H^{1}\right),
$$

from where, using interpolation, we deduce that

$$
\left(f_{n}\right)_{n} \text { is uniformly bounded in } H^{1-\vartheta}\left([0, T) ; H^{1+\vartheta(\varepsilon+1)}\right), \vartheta \in[0,1] .
$$

Setting

$$
\vartheta \in\left[\frac{1}{2(\varepsilon+1)}, \frac{1}{2}\right)
$$

we deduce that

$$
\left(f_{n}\right)_{n} \text { is uniformly bounded in } C^{0, \frac{1}{2}-\vartheta}\left([0, T) ; H^{1+\vartheta(\varepsilon+1)}\right) .
$$

We obtain that $\left(f_{n}\right)_{n}$ is equicontinuous-in-time. We can hence apply Ascoli-Arzelá theorem in order to deduce that there exists an $f \in C^{0, \frac{1}{2}-\vartheta}\left([0, T) ; H^{1+\vartheta(\varepsilon+1)}\right)$ such that, taking a subsequence if necessary, $f_{n} \rightarrow f$ in $C^{0, \frac{1}{2}-\vartheta}\left([0, T) ; H^{1+\vartheta(\varepsilon+1)}\right)$ as long as $\vartheta$ satisfies (3.42). It is a classical argument to prove that $f$ solves (1.3) and that it is unique.

\section{Gravity-capillarity driven system (1.1).}

\subsection{Proof of Theorem 2.5.}

Step 1: A priori estimates. We only prove the a priori estimates, since the approximation procedure is standard and it can be done using a Galerkin scheme. We have that

$$
\frac{\mathrm{d}}{\mathrm{d} t}\|f(t)\|_{\dot{A}^{1}}+\nu\|f(t)\|_{A^{4}}+\|f(t)\|_{A^{2}} \leqslant\left\|\partial_{x}^{2}\left(\llbracket \mathcal{H}, f \rrbracket\left(\nu \Lambda^{3} f+\Lambda f\right)\right)\right\|_{A^{0}} .
$$

As before,

$$
\left\|\partial_{x}^{2}(\llbracket \mathcal{H}, f \rrbracket \Lambda f)\right\|_{A^{0}} \leqslant 2\|f\|_{A^{1}}\|f\|_{A^{2}} .
$$

In Fourier variables, we have that

$$
\begin{aligned}
\partial_{x}^{2}\left(\llbracket \widehat{\llbracket \mathcal{H}, f \rrbracket} \Lambda^{3} f\right) & =\partial_{x} \Lambda\left(f \Lambda^{3} \widehat{f)-} \partial_{x}^{2}\left(f \partial_{x}^{3} f\right)\right. \\
& =\hat{f}(k-m) \hat{f}(m) p(k, m)
\end{aligned}
$$

Copyright $@$ by SIAM. Unauthorized reproduction of this article is prohibited. 
with

$$
p(k, m)=i k\left(|k||k-m|^{3}-k(k-m)^{3}\right)=i k|k||k-m|^{3}\left(1-\frac{k(k-m)^{3}}{|k||k-m|^{3}}\right) .
$$

Again $p \neq 0$ if and only if $0<|k|<|m|$. We find that

$$
|p(k, m)| \leqslant 2|k||k||k-m|^{3} \leq 2|m|^{2}|k-m|^{3} .
$$

Thus, using the interpolation inequality (valid for $0 \leq r \leq 3$ )

$$
\|u\|_{A^{r}} \leqslant\|u\|_{A^{0}}^{1-r / 3}\|u\|_{A^{3}}^{r / 3},
$$

we find that

$$
\left\|\partial_{x}^{2}\left(\llbracket \mathcal{H}, f \rrbracket \Lambda^{3} f\right)\right\|_{A^{0}} \leqslant 2 \nu\|f\|_{A^{1}}\|f\|_{A^{4}} .
$$

As a consequence, we find the inequality

$$
\frac{\mathrm{d}}{\mathrm{d} t}\|f(t)\|_{\dot{A}^{1}}+\nu\|f(t)\|_{A^{4}}+\|f(t)\|_{A^{2}} \leqslant 2\|f\|_{A^{1}}\|f\|_{A^{2}}+2 \nu\|f\|_{A^{1}}\|f\|_{A^{4}},
$$

and we conclude the result.

Step 2: Decay. We have that

$$
\frac{\mathrm{d}}{\mathrm{d} t}\|f(t)\|_{A^{0}}+\nu\|f(t)\|_{A^{3}}+\|f(t)\|_{A^{1}} \leqslant 2\|f\|_{A^{1}}^{2}+2 \nu\|f\|_{A^{1}}\|f\|_{A^{3}} ;
$$

thus,

$$
\frac{\mathrm{d}}{\mathrm{d} t}\|f(t)\|_{A^{0}}+\left(\nu\|f(t)\|_{A^{3}}+\|f(t)\|_{A^{1}}\right)\left(1-2\|f\|_{A^{1}}\right) \leqslant 0 .
$$

From here we conclude using a Poincaré-type inequality.

\subsection{Proof of Theorem 2.7.}

\section{Step 1: A priori estimates.}

Step 1.1: $\boldsymbol{L}^{\mathbf{2}}$ estimates. For the sake of clarity, we first perform $L^{2}$ energy estimates for (1.1), and we study the particular commutation properties of the nonlinearity of (1.1). We multiply (1.1) by $f$ and integrate in $\mathbb{T}$ to deduce the following:

$$
\frac{1}{2} \frac{\mathrm{d}}{\mathrm{d} t}\|f(t)\|_{L^{2}}^{2}+\nu\left\|\Lambda^{3 / 2} f(t)\right\|_{L^{2}}^{2}+\left\|\Lambda^{1 / 2} f(t)\right\|_{L^{2}}^{2}=\mathcal{N}(f, f, f)+\mathcal{M}(f, f, f),
$$

where $\mathcal{N}$ is defined in (3.9) and $\mathcal{M}$ is defined as

$$
\mathcal{M}\left(g_{1}, g_{2}, g_{3}\right)=\nu \int_{\mathbb{T}} g_{1}\left(\Lambda^{3} g_{2} \Lambda g_{3}+\partial_{x}^{3} g_{2} \partial_{x} g_{3}\right) \mathrm{d} x .
$$

Our goal now is to prove that

$$
|\mathcal{M}(g, h, h)| \leqslant C \nu\|g\|_{H^{2}(\mathbb{T})}\|h\|_{H^{1 / 2}(\mathbb{T})}^{1 / 2}\|h\|_{H^{3 / 2}(\mathbb{T})}^{3 / 2} .
$$

Indeed, using Plancherel's theorem, we can write

$$
\mathcal{M}(g, h, h)=\nu \sum_{n, k} \overline{\hat{g}}_{n} \hat{h}_{n-k} \hat{h}_{k}(n-k)^{3} k(\operatorname{sgn}(n-k) \operatorname{sgn} k+1) .
$$

Copyright $@$ by SIAM. Unauthorized reproduction of this article is prohibited. 
As was done in the proof of (3.10) we observe that the nonzero contributions arise in the set

$$
0<k<n \quad \text { or } \quad n<k<0 .
$$

Thus, taking advantage of this symmetry,

$$
\mathcal{M}(g, h, h)=4 \nu \sum_{0<k<n}(n-k)^{3} k \operatorname{Re}\left(\overline{\hat{g}}_{n} \hat{h}_{n-k} \hat{h}_{k}\right),
$$

which entails the inequality

$$
|\mathcal{M}(g, h, h)| \leqslant 4 \sum_{0<k<n}(n-k)^{3} k\left|\hat{g}_{n}\right|\left|\hat{h}_{n-k}\right|\left|\hat{h}_{k}\right| .
$$

Since the summation set is localized in the Fourier modes $0<k<n$ we can deduce the inequality

$$
(n-k)^{3} k<n^{2}(n-k) k ;
$$

thus, we can estimate as follows:

$$
\begin{aligned}
|\mathcal{M}(g, h, h)| & \leqslant C\|g\|_{H^{2}}\|h\|_{\dot{W}^{1,4}}^{2} \\
& \leqslant C\|g\|_{H^{2}}\|h\|_{H^{5 / 4}}^{2} \\
& \leqslant C\|g\|_{H^{2}}\|h\|_{H^{1}}\|h\|_{H^{3 / 2}} \\
& \leqslant C\|g\|_{H^{2}}\|h\|_{H^{1 / 2}}^{1 / 2}\|h\|_{H^{3 / 2}}^{3 / 2} .
\end{aligned}
$$

Equipped with (4.2), we can, hence, conclude the proof of the $L^{2}$ estimates. In fact using Young's inequality we deduce that

$$
|\mathcal{M}(f, f, f)| \leqslant \alpha\|f\|_{H^{1 / 2}}^{2}+\frac{C \nu^{4 / 3}}{\alpha}\|f\|_{H^{2}}^{4 / 3}\|f\|_{H^{3 / 2}}^{2} .
$$

Using (3.10) and the continuous embedding of $H^{2}$ into $H^{3 / 2}$ we obtain that

$$
\begin{aligned}
|\mathcal{N}(f, f, f)| & \leqslant C\|f\|_{H^{3 / 2}}\|f\|_{H^{1 / 2}}^{2} \\
& \leqslant C\|f\|_{H^{2}}\|f\|_{H^{1 / 2}}^{2}
\end{aligned}
$$

From here, taking $\alpha=1 / 4$ and if

$$
\|f(t)\|_{H^{2}} \leqslant \frac{1}{C} \min \left\{1, \nu^{-\frac{1}{4}}\right\} \quad \forall t \in[0, T],
$$

we deduce that

$$
|\mathcal{N}(f, f, f)|+|\mathcal{M}(f, f, f)| \leqslant \frac{\nu}{2}\|f\|_{H^{3 / 2}}^{2}+\frac{1}{2}\|f\|_{H^{1 / 2}}^{2}
$$

leads us to

$$
\frac{\mathrm{d}}{\mathrm{d} t}\|f(t)\|_{L^{2}}^{2}+\int_{0}^{t}\left(\nu\left\|\Lambda^{3 / 2} f(s)\right\|_{L^{2}}^{2}+\left\|\Lambda^{1 / 2} f(s)\right\|_{L^{2}}^{2}\right) \mathrm{d} s \leqslant\left\|f_{0}\right\|_{L^{2}}^{2} .
$$

Copyright (c) by SIAM. Unauthorized reproduction of this article is prohibited. 
Step 1.2: $\boldsymbol{H}^{2}$ estimates. We want now to prove global $H^{2}$ estimates for (1.1) stemming from small initial data. We apply the dyadic truncation $\triangle_{q}$ to the left of (1.1); we multiply the resulting equation for $\triangle_{q} f$ and integrate in $x$ obtaining

$$
\frac{1}{2} \frac{\mathrm{d}}{\mathrm{d} t}\left\|\triangle_{q} f\right\|_{L^{2}}^{2}+\nu\left\|\triangle_{q} \Lambda^{3 / 2} f\right\|_{L^{2}}^{2}+\left\|\triangle_{q} \Lambda^{1 / 2} f\right\|_{L^{2}}^{2}=A_{q}-B_{q}+C_{q}+D_{q},
$$

where

$$
\begin{gathered}
A_{q}=\int \triangle_{q}(f \Lambda f) \triangle_{q} \Lambda f \mathrm{~d} x, \\
B_{q}=\int \triangle_{q}\left(f \partial_{x} f\right) \triangle_{q} \partial_{x} f \mathrm{~d} x, \\
C_{q}=\nu \int \triangle_{q}\left(f \Lambda^{3} f\right) \triangle_{q} \Lambda f \mathrm{~d} x, \\
D_{q}=\int \triangle_{q}\left(f \partial_{x}^{3} f\right) \triangle_{q} \partial_{x} f \mathrm{~d} x .
\end{gathered}
$$

We can use the estimates performed before (see in particular the right-hand side of (3.29)) in order to deduce

$$
\left|A_{q}-B_{q}\right| \leqslant C b_{q} 2^{-4 q}\|f\|_{H^{2}}\left\|\Lambda^{1 / 2} f\right\|_{H^{2}}^{2}
$$

We can hence now focus on the purely nonlinear part which is characteristic of (1.1) when $\nu>0$, i.e., $-\nu\left(C_{q}+D_{q}\right)$. We can use Bony decomposition A.2 in order to decompose $C_{q}$ and $D_{q}$ as

$$
\begin{aligned}
& C_{q}=T_{1, q}^{C}+T_{2, q}^{C}+T_{3, q}^{C}+R_{q}^{C}, \\
& D_{q}=T_{1, q}^{D}+T_{2, q}^{D}+T_{3, q}^{D}+R_{q}^{D},
\end{aligned}
$$

where

$$
\begin{aligned}
T_{1, q}^{C} & =\int S_{q-1} f \triangle_{q} \Lambda^{3} f \triangle_{q} \Lambda f \mathrm{~d} x, \\
T_{1, q}^{D} & =\int S_{q-1} f \triangle_{q} \partial_{x}^{3} f \triangle_{q} \partial_{x} f \mathrm{~d} x, \\
T_{2, q}^{C} & =\sum_{\left|q-q^{\prime}\right| \leqslant 4} \int\left[\triangle_{q}, S_{q^{\prime}-1} f\right] \triangle_{q^{\prime}} \Lambda^{3} f \triangle_{q} \Lambda f \mathrm{~d} x, \\
T_{2, q}^{D} & =\sum_{\left|q-q^{\prime}\right| \leqslant 4} \int\left[\triangle_{q}, S_{q^{\prime}-1} f\right] \triangle_{q^{\prime}} \partial_{x}^{3} f \triangle_{q} \partial_{x} f \mathrm{~d} x, \\
T_{3, q}^{C} & =\sum_{\left|q-q^{\prime}\right| \leqslant 4} \int\left(S_{q^{\prime}-1}-S_{q}\right) f \triangle_{q} \triangle_{q^{\prime}} \Lambda^{3} f \triangle_{q} \Lambda f \mathrm{~d} x, \\
T_{3, q}^{D} & =\sum_{\left|q-q^{\prime}\right| \leqslant 4} \int\left(S_{q^{\prime}-1}-S_{q}\right) f \triangle_{q} \triangle_{q^{\prime}} \partial_{x}^{3} f \triangle_{q} \partial_{x} f \mathrm{~d} x, \\
R_{q}^{C} & =\sum_{q^{\prime}>q-4} \int \triangle_{q}\left(\triangle_{q^{\prime}-1} f S_{q^{\prime}+2} \Lambda^{3} f\right) \triangle_{q} \Lambda f \mathrm{~d} x, \\
R_{q}^{D} & =\sum_{q^{\prime}>q-4} \int \triangle_{q}\left(\triangle_{q^{\prime}-1} f S_{q^{\prime}+2} \partial_{x}^{3} f\right) \triangle_{q} \partial_{x} f \mathrm{~d} x .
\end{aligned}
$$

Copyright $@$ by SIAM. Unauthorized reproduction of this article is prohibited. 
We remark now that

$$
-\nu\left(T_{1, q}^{C}+T_{1, q}^{D}\right)=\mathcal{M}\left(S_{q-1} f, \triangle_{q} f . \triangle_{q} f\right) .
$$

We can apply the estimate (4.2) and (A.3) in order to deduce the bound

$$
\begin{aligned}
\left|\mathcal{M}\left(S_{q-1} f, \triangle_{q} f . \triangle_{q} f\right)\right| & \leqslant C \nu\|f\|_{H^{2}}\left\|\triangle_{q} \Lambda^{1 / 2} f\right\|_{L^{2}}^{1 / 2}\left\|\triangle_{q} \Lambda^{3 / 2} f\right\|_{L^{2}}^{3 / 2} \\
& \leqslant C b_{q} 2^{-4 q} \nu\|f\|_{H^{2}}\left\|\Lambda^{1 / 2} f\right\|_{H^{2}}^{1 / 2}\left\|\Lambda^{3 / 2} f\right\|_{H^{2}}^{3 / 2} .
\end{aligned}
$$

Next we focus on the remainder terms. Using the Hölder inequality, Sobolev embeddings, (A.3), and interpolation of Sobolev spaces it is possible to deduce the following estimate:

$$
\begin{aligned}
\left|R_{q}^{C}\right| & \leqslant C \sum_{q^{\prime}>q-4}\left\|S_{q^{\prime}+2} \Lambda^{3} f\right\|_{L^{2}}\left\|\triangle_{q^{\prime}-1} f\right\|_{L^{4}}\left\|\triangle_{q} \Lambda f\right\|_{L^{4}} \\
& \leqslant C \sum_{q^{\prime}>q-4}\left\|S_{q^{\prime}+2} \Lambda^{2} f\right\|_{L^{2}}\left\|\triangle_{q^{\prime}-1} \Lambda f\right\|_{L^{4}}\left\|\triangle_{q} \Lambda f\right\|_{L^{4}} \\
& \leqslant C c_{q} 2^{-4 q}\|f\|_{H^{2}}\left\|\Lambda^{5 / 4} f\right\|_{H^{2}}^{2} \sum_{q^{\prime}>q-4} 2^{2\left(q-q^{\prime}\right)} c_{q^{\prime}} \\
& \leqslant C b_{q} 2^{-4 q}\|f\|_{H^{2}}\left\|\Lambda^{5 / 4} f\right\|_{H^{2}}^{2} \\
& \leqslant C b_{q} 2^{-4 q}\|f\|_{H^{2}}\left\|\Lambda^{1 / 2} f\right\|_{H^{2}}^{1 / 2}\left\|\Lambda^{3 / 2} f\right\|_{H^{2}}^{3 / 2}
\end{aligned}
$$

In the above estimates we have that

$$
b_{q}=c_{q}\left(\left(1_{p<4} 2^{2 q^{\prime}}\right) \star_{q^{\prime}} c_{q^{\prime}}\right)_{q}
$$

which is $\ell^{2}$ as long as $\left(c_{q}\right)_{q} \in \ell^{1}$. Similar computations holds for the term $R_{q}^{D}$, from where we deduce that

$$
\left|R_{q}^{D}\right| \leqslant C b_{q} 2^{-4 q}\|f\|_{H^{2}}\left\|\Lambda^{1 / 2} f\right\|_{H^{2}}^{1 / 2}\left\|\Lambda^{3 / 2} f\right\|_{H^{2}}^{3 / 2} .
$$

Next we study the term $T_{2, q}^{C}$. Using Lemma A.1 we obtain

$$
\left|T_{2, q}^{C}\right| \leqslant \sum_{\left|q-q^{\prime}\right| \leqslant 4} 2^{-q}\|\Lambda f\|_{L^{\infty}}\left\|\triangle_{q^{\prime}} \Lambda^{3} f\right\|_{L^{2}}\left\|\triangle_{q} \Lambda f\right\|_{L^{2}} .
$$

Now, since $\left\|\triangle_{q^{\prime}} \Lambda^{3} f\right\|_{L^{2}} \lesssim 2^{\frac{3 q^{\prime}}{2}}\left\|\triangle_{q^{\prime}} \Lambda^{3 / 2} f\right\|_{L^{2}}$ and as a consequence of $2^{q^{\prime}} \sim 2^{q}$ for $\left|q-q^{\prime}\right| \leqslant 4$ we find that

$$
\begin{aligned}
\left|T_{2, q}^{C}\right| & \leqslant C \sum_{\left|q-q^{\prime}\right| \leqslant 4}\|f\|_{H^{2}}\left\|\triangle_{q^{\prime}} \Lambda^{3 / 2} f\right\|_{L^{2}}\left\|\triangle_{q} \Lambda^{3 / 2} f\right\|_{L^{2}} \\
& \leqslant C b_{q} 2^{-4 q}\|f\|_{H^{2}}\left\|\Lambda^{3 / 2} f\right\|_{H^{2}}^{2} .
\end{aligned}
$$

We can apply the very same computations to $T_{2, q}^{D}$, which give

$$
\left|T_{2, q}^{D}\right| \leqslant C b_{q} 2^{-4 q}\|f\|_{H^{2}}\left\|\Lambda^{3 / 2} f\right\|_{H^{2}}^{2} .
$$

Copyright (c) by SIAM. Unauthorized reproduction of this article is prohibited. 
The terms $T_{3, q}^{C}, T_{3, q}^{D}$ enjoy analog bounds and are the overall more regular terms, being composed by elements localized in dyadic annuli; thus

$$
\text { (4.7) } \nu\left|C_{q}+D_{q}\right| \leqslant C \nu b_{q} 2^{-4 q}\left[\|f\|_{H^{2}}\left\|\Lambda^{1 / 2} f\right\|_{H^{2}}^{1 / 2}\left\|\Lambda^{3 / 2} f\right\|_{H^{2}}^{3 / 2}+\|f\|_{H^{2}}\left\|\Lambda^{3 / 2} f\right\|_{H^{2}}^{2}\right] \text {. }
$$

Collecting (4.6) and (4.7) in (4.5), multiplying the resulting inequality for $2^{4 q}$, summing up in $q \in \mathbb{Z}$, and applying Young's convexity inequality we conclude the following inequality:

$$
\begin{aligned}
\frac{1}{2} \frac{\mathrm{d}}{\mathrm{d} t}\|f\|_{H^{2}}^{2}+\nu\left\|\Lambda^{3 / 2} f\right\|_{H^{2}}^{2}+\left\|\Lambda^{1 / 2} f\right\|_{H^{2}}^{2} & \leqslant \\
& \left(\frac{1}{4}+C\|f\|_{H^{2}}\right)\left\|\Lambda^{1 / 2} f\right\|_{H^{2}}^{2} \\
& +C\left(\nu\|f\|_{H^{2}}+\nu^{\frac{4}{3}}\|f\|_{H^{2}}^{\frac{4}{3}}\right)\left\|\Lambda^{3 / 2} f\right\|_{H^{2}}^{2}
\end{aligned}
$$

From the previous inequality, if the initial data satisfies

$$
\left\|f_{0}\right\|_{H^{2}(\mathbb{T})} \leqslant \frac{1}{C} \min \left\{1, \nu^{-\frac{1}{4}}\right\},
$$

we obtain the desired global bound

$$
\|f(t)\|_{H^{2}(\mathbb{T})}^{2}+\int_{0}^{t}\left[\nu\left\|\Lambda^{3 / 2} f(s)\right\|_{H^{2}(\mathbb{T})}^{2}+\left\|\Lambda^{1 / 2} f(s)\right\|_{H^{2}(\mathbb{T})}^{2}\right] \mathrm{d} s \leqslant\left\|f_{0}\right\|_{H^{2}(\mathbb{T})}^{2} .
$$

Step 2: Approximated solutions and passing to the limit. The proof is again a standard approximation argument. Using the projection operator defined in (3.41), we consider the approximated problems

$$
\left\{\begin{array}{l}
\partial_{t} f_{n}+\nu \Lambda^{3} f_{n}+\Lambda f_{n} \\
=-\mathcal{J}_{n}\left[\nu\left[\Lambda\left(f_{n} \Lambda^{3} f_{n}\right)-\partial_{x}\left(f_{n} \partial_{x}^{3} f_{n}\right)\right]+\left[\Lambda\left(f_{n} \Lambda f_{n}\right)+\partial_{x}\left(f_{n} \partial_{x} f_{n}\right)\right]\right] \\
\left.f_{n}\right|_{t=0}=\mathcal{J}_{n} f_{0}
\end{array}\right.
$$

We can now define the space

$$
\widetilde{\mathcal{H}}_{n}=\left\{u \in \mathcal{D}^{\prime} \mid u \in H^{2}, \operatorname{supp} \hat{u} \subset B_{n}(0)\right\} .
$$

Using the Cauchy-Lipschitz theorem and the estimate (4.9) we deduce that if $f_{0}$ satisfies the smallness hypothesis (4.8), then $f_{n} \in C^{1}\left(\mathbb{R}_{+} ; \widetilde{\mathcal{H}}_{n}\right) \cap L^{2}\left(\mathbb{R}_{+} ; H^{\frac{7}{2}}\right)$. Moreover $\left(\partial_{t} f_{n}\right)_{n}$ is uniformly bounded in $L^{2}\left(\mathbb{R}_{+} ; H^{-N}\right)$ for $N$ sufficiently large, and invoking the Aubin-Lions lemma we deduce that

$$
\left(f_{n}\right)_{n} \text { is compact in } L^{2}\left([0, T] ; H^{\frac{7}{2}-\epsilon}\right) \forall \epsilon, T>0 .
$$

Passing to a subsequence if necessary we obtain the convergence

$$
f_{n} \rightarrow f \text { in } L^{2}\left([0, T] ; H^{\frac{7}{2}-\epsilon}\right)
$$

and the limit element $f$ satisfies the energy inequality (4.9), so

$$
f \in C\left(\mathbb{R}_{+} ; H^{2}\right) \cap L^{2}\left(\mathbb{R}_{+} ; H^{\frac{7}{2}}\right) .
$$

Copyright $\odot$ by SIAM. Unauthorized reproduction of this article is prohibited. 


\section{Appendix A. Elements of Littlewood-Paley theory.}

A tool that is widely used all along the paper is the theory of Littlewood-Paley, which consists in doing a dyadic cutoff of the frequencies. Let us define the (homogeneous) truncation operators as follows:

$$
\triangle_{q} u=\sum_{n \in \mathbb{Z}^{3}} \hat{u}_{n} \varphi\left(\frac{|\check{n}|}{2^{q}}\right) e^{i \check{n} \cdot x} \quad \text { for } q \in \mathbb{Z},
$$

where $u \in \mathcal{D}^{\prime}\left(\mathbb{T}^{3}\right)$ and $\hat{u}_{n}$ are the Fourier coefficients of $u$. The function $\varphi$ is a smooth function with compact support such that

$$
\operatorname{supp} \varphi \subset \mathcal{C}\left(\frac{3}{4}, \frac{8}{3}\right)
$$

and such that for all $t \in \mathbb{R}$,

$$
\sum_{q \in \mathbb{Z}} \varphi\left(2^{-q} t\right)=1
$$

Let us define further the low frequencies cutoff operator

$$
S_{q} u=\sum_{q^{\prime} \leqslant q-1} \triangle_{q^{\prime}} u
$$

The dyadic decomposition turns out to be very useful also when it comes to study the product between two distributions. We can in fact, at least formally, write for two distributions $u$ and $v$

$$
u=\sum_{q \in \mathbb{Z}} \triangle_{q} u, \quad v=\sum_{q^{\prime} \in \mathbb{Z}} \triangle_{q^{\prime}} v, \quad u \cdot v=\sum_{\substack{q \in \mathbb{Z} \\ q^{\prime} \in \mathbb{Z}}} \triangle_{q} u \cdot \triangle_{q^{\prime}} v .
$$

Paradifferential calculus is a mathematical tool for splitting the above sum in three parts:

$$
u \cdot v=T_{u} v+T_{v} u+R(u, v),
$$

where

$$
T_{u} v=\sum_{q} S_{q-1} u \triangle_{q} v, \quad T_{v} u=\sum_{q^{\prime}} S_{q^{\prime}-1} v \triangle_{q^{\prime}} u, \quad R(u, v)=\sum_{k} \sum_{|\nu| \leqslant 1} \triangle_{k} u \triangle_{k+\nu} v .
$$

The following almost orthogonality properties hold:

$$
\begin{aligned}
\triangle_{q}\left(S_{q} a \triangle_{q^{\prime}} b\right) & =0 & & \text { if }\left|q-q^{\prime}\right| \geqslant 5, \\
\triangle_{q}\left(\triangle_{q^{\prime}} a \triangle_{q^{\prime}+\nu} b\right) & =0 & & \text { if } q^{\prime}<q-4,|\nu| \leqslant 1,
\end{aligned}
$$

and hence we will often use the following relation:

$$
\begin{aligned}
\triangle_{q}(u \cdot v)= & \sum_{\left|q-q^{\prime}\right| \leqslant 4} \triangle_{q}\left(S_{q^{\prime}-1} v \triangle_{q^{\prime}} u\right)+\sum_{\left|q-q^{\prime}\right| \leqslant 4} \triangle_{q}\left(S_{q^{\prime}-1} u \triangle_{q^{\prime}} v\right) \\
& +\sum_{q^{\prime} \geqslant q-4} \sum_{|\nu| \leqslant 1} \triangle_{q}\left(\triangle_{q^{\prime}} a \triangle_{q^{\prime}+\nu} b\right) \\
= & \sum_{\left|q-q^{\prime}\right| \leqslant 4} \triangle_{q}\left(S_{q^{\prime}-1} v \triangle_{q^{\prime}} u\right)+\sum_{q^{\prime}>q-4} \triangle_{q}\left(S_{q^{\prime}+2} u \triangle_{q^{\prime}} v\right) .
\end{aligned}
$$

Copyright (c) by SIAM. Unauthorized reproduction of this article is prohibited. 
In the paper [12] J.-Y. Chemin and N. Lerner introduced the following decomposition which will be very useful in our context:

$$
\begin{aligned}
\triangle_{q}(u v)= & S_{q-1} u \triangle_{q} v \\
& +\sum_{\left|q-q^{\prime}\right| \leqslant 4}\left\{\left[\triangle_{q}, S_{q^{\prime}-1} u\right] \triangle_{q^{\prime}} v+\left(S_{q^{\prime}-1} u-S_{q} u\right) \triangle_{q} \triangle_{q^{\prime}} v\right\} \\
& +\sum_{q^{\prime}>q-4} \triangle_{q}\left(S_{q^{\prime}+2} v \triangle_{q^{\prime}} u\right),
\end{aligned}
$$

where the commutator $\left[\triangle_{q}, a\right] b$ is defined as

$$
\left[\triangle_{q}, a\right] b=\triangle_{q}(a b)-a \triangle_{q} b .
$$

There is an interesting relation of regularity between dyadic blocks and full function in the Sobolev spaces, i.e.,

$$
\left\|\triangle_{q} f\right\|_{L^{p}(\mathbb{T})} \leqslant C c_{q}^{(p)} 2^{-q s}\|f\|_{W^{s, p}(\mathbb{T})}
$$

with $\left\|\left\{c_{q}^{(p)}\right\}_{q \in \mathbb{Z}}\right\|_{\ell^{p}(\mathbb{Z})} \equiv 1$; if $p=2$ we denote $\left\{c_{q}^{(2)}\right\}_{q}=\left\{c_{q}\right\}_{q}$ for simplicity. In the same way we denote as $b_{q}$ a sequence in $\ell^{1}(\mathbb{Z})$ such that $\sum_{q}\left|b_{q}\right| \leqslant 1$.

Finally we state a lemma that shows that the commutator with the dyadic block in the vertical frequencies is a regularizing operator. The proof of this lemma can be found in $[6$, Lemma 2.97 , page 110$]$ for the whole space $\mathbb{R}^{d}$; the proof for the periodic space $\mathbb{T}^{d}$ is analogous.

Lemma A.1. Let $\mathbb{T}^{d}$ be a d-dimensional torus and $p, r, s$ real positive numbers such that $p, r, s \in[1, \infty]$ and $\frac{1}{p}=\frac{1}{r}+\frac{1}{s}$. There exists a constant $C$ such that for all vector fields $u$ and $v$ on $\mathbb{T}^{d}$ we have the inequality

$$
\left\|\left[\triangle_{q}, u\right] v\right\|_{L^{p}} \leqslant C 2^{-q}\|\nabla u\|_{L^{r}}\|v\|_{L^{s}} .
$$

\section{REFERENCES}

[1] T. Alazard, Convexity and the Hele-Shaw Equation, Water Waves, 2020, in press.

[2] T. Alazard And D. Bresch, Functional Inequalities and Strong Lyapunov Functionals for Free Surface Flows in Fluid Dynamics, preprint, arXiv:2004.03440.

[3] T. Alazard And J.-M. Delort, Sobolev estimates for two dimensional gravity water waves, Astérisque, 374 (2015), pp. 1-241

[4] T. Alazard and O. Lazar, Paralinearization of the Muskat equation and application to the Cauchy problem, Arch. Ration. Mech. Anal., 237 (2020), pp. 545-583.

[5] T. Alazard, N. Meunier, And D. Smets, Lyapunov functions, identities and the Cauchy problem for the Hele-Shaw equation, Comm. Math. Phys., 377 (2020), pp. 1421-1459.

[6] H. Bahouri, J.-Y. Chemin, and R. Danchin, Fourier Analysis and Nonlinear Partial Differential Equations, Grundlehren Math. Wiss. 343, Springer, Heidelberg, 2011.

[7] L. C. Berselli, D. Córdoba, and R. Granero-Belinchón, Local solvability and turning for the inhomogeneous Muskat problem, Interfaces Free Bound., 16 (2014), pp. 175-213.

[8] J. BoussinesQ, Théorie des ondes et des remous qui se propagent le long d'un canal rectangulaire horizontal, en communiquant au liquide contenu dans ce canal des vitesses sensiblement pareilles de la surface au fond, J. Math. Pures Appl. 17 (1872), pp. 55-108.

[9] S. Cameron, Global well-posedness for the 2D Muskat problem with slope less than 1, Anal. PDE, 12 (2017), pp. 997-1022.

[10] A. Castro, D. Córdoba, C. Fefferman, F. Gancedo, and M. Lopez-Fernandez, RayleighTaylor breakdown for the Muskat problem with applications to water waves, Ann. of Math. (2), 175 (2012), pp. 909-948.

Copyright (c) by SIAM. Unauthorized reproduction of this article is prohibited. 
[11] A. Castro, D. Córdoba, C. Fefferman, and F. Gancedo, Splash singularities for the one-phase Muskat problem in stable regimes, Arch. Ration. Mech. Anal., 222 (2016), pp. $213-243$.

[12] J.-Y. Chemin And N. Lerner, Flot de champs de vecteurs non lipschitziens et équations de Navier-Stokes, J. Differential Equations, 121 (1995), pp. 314-328.

[13] C.-H. A. Cheng, R. Granero-Belinchón, and S. Shkoller, Well-posedness of the Muskat problem with $H^{2}$ initial data, Adv. Math., 286 (2016), pp. 32-104.

[14] C.-H. A. Cheng, R. Granero-Belinchón, S. Shkoller, and J. Wilkening, Rigorous Asymptotic models of water waves, Water Waves, 1 (2019), pp. 71-130.

[15] P. Constantin, D. Cordoba, F. Gancedo, and R. M. Strain, On the global existence for the Muskat problem, J. Eur. Math. Soc. (JEMS), 15 (2013), pp. 201-227.

[16] P. Constantin, F. Gancedo, R. Shvydkoy, and V. Vicol, Global regularity for $2 D$ Muskat equations with finite slope, Ann. Inst. H. Poincaré Anal. Non Linéaire, 34 (2016), pp. 1041-1074.

[17] A. Cordoba, D. Córdoba, And F. Gancedo, Interface evolution: The Hele-Shaw and Muskat problems, Ann. of Math. (2), 173 (2011), pp. 477-542.

[18] D. Cóndoba And F. Gancedo, Contour dynamics of incompressible 3-D fluids in a porous medium with different densities, Comm. Math. Phys., 273 (2007), pp. 445-471.

[19] D. Córdoba and F. Gancedo, A maximum principle for the Muskat problem for fluids with different densities, Comm. Math. Phys., 286 (2009), pp. 681-696.

[20] D. Córdoba, F. Gancedo, and R. Orive, A note on interface dynamics for convection in porous media, Phys. D, 237 (2008), pp. 1488-1497.

[21] D. Córdoba, J. Gómez-Serrano, and A. Zlatoš, A note on stability shifting for the Muskat problem, Philos. Trans. Roy. Soc. A, 373 (2015), 20140278.

[22] D. Córdoba, J. Gómez-Serrano, and A. Zlatoš, A note on stability shifting for the Muskat problem, II: From stable to unstable and back to stable, Anal. PDE, 10 (2017), pp. 367-378.

[23] D. Córdoba, R. Granero-Belinchón, and R. Orive, On the confined Muskat problem: Differences with the deep water regime, Comm. Math. Sci., 12 (2014), pp. 423-455.

[24] D. Cordoba and T. Pernas-Castaño, Non-splat singularity for the one-phase Muskat problem, Trans. Amer. Math. Soc., 369 (2017), pp. 711-754.

[25] D. Cordoba and O. Lazar, Global Well-posedness for the $2 D$ Stable Muskat Problem in $H^{3 / 2}$, preprint, arXiv:1803.07528, 2018.

[26] D. Coutand And S. ShKoller, On the impossibility of finite-time splash singularities for vortex sheets, Arch. Ration. Mech. Anal., 221 (2016), pp. 987-1033.

[27] W. Craig, An existence theory for water waves and the Boussinesq and Korteweg-deVries scaling limits, Comm. Partial Differential Equations, 10 (1985), pp. 787-1003.

[28] W. Craig and C. Sulem, Numerical simulation of gravity waves, J. Comput. Phys., 108 (1993), pp. $73-83$.

[29] W. Craig, U. Schanz, AND C. Sulem, The modulational regime of three-dimensional water waves and the Davey-Stewartson system, Ann. Inst. H. Poincaré Anal. Non Linéaire, 14 (1997), pp. 615-667.

[30] C. Fefferman, A. D. Ionescu, And V. Lie, On the absence of splash singularities in the case of two-fluid interfaces, Duke Math. J., 165 (2016), pp. 417-462.

[31] F. Gancedo and O. Lazar, Global Well-posedness for the 3D Muskat Problem in the Critical Sobolev Space, preprint, arXiv:2006.01787, 2020.

[32] F. Gancedo and R. M Strain, Absence of splash singularities for surface quasi-geostrophic sharp fronts and the Muskat problem, Proc. Natl. Acad. Sci. USA, 111 (2014), pp. 635-639.

[33] J. Gómez-Serrano and R. Granero-Belinchón, On turning waves for the inhomogeneous Muskat problem: A computer-assisted proof, Nonlinearity, 27 (2014), pp. 1471-1498.

[34] R. Granero-Belinchón, Global existence for the confined Muskat problem, SIAM J. Math. Anal., 46 (2014), pp. 1651-1680.

[35] F. Gancedo, R. Granero-Belinchón, and S. Scrobogna, Surface tension stabilization of the Rayleigh-Taylor instability for a fluid layer in a porous medium, Ann. Inst. H. Poincaré Anal Non Linéaire, in press.

[36] R. Granero-Belinchón and O. Lazar, Growth in the Muskat problem, Math. Model. Nat. Phenom., 15 (2020), 7.

[37] R. Granero-Belinchón and S. Scrobogna, Asymptotic models for free boundary flow in porous media, Phys. D, 392 (2019), pp. 1-16.

[38] R. Granero-Belinchón and S. Scrobogna, Well-posedness of a water wave model with viscous effects, Proc. Amer. Math. Soc., in press.

[39] R. Granero-Belinchón and S. Scrobogna, Models for damped water waves, SIAM J. Appl. Math., 79 (2019), pp. 2530-2550.

Copyright $@$ by SIAM. Unauthorized reproduction of this article is prohibited. 
[40] R. Granero-Belinchón and S. Shkoller, Well-posedness and decay to equilibrium for the Muskat problem with discontinuous permeability, Trans. Amer. Math. Soc., 372 (2019), pp. $2255-2286$.

[41] R. Granero-Belinchón and S. Shkoller, A model for Rayleigh-Taylor mixing and interface turnover, Multiscale Model. Simul., 15 (2017), pp. 274-308.

[42] R. Granero-Belinchón, G. Navarro, and A. Ortega, On the effect of boundaries in twophase porous flow, Nonlinearity, 28 (2015), pp. 435-461.

[43] D. Lannes, The Water Waves Problem: Mathematical Analysis and Asymptotics, American Mathematical Society, Providence, RI, 2013.

[44] M. Muskat, Two fluid systems in porous media. The encroachment of water into an oil sand, Physics, 5 (1934), pp. 250-264.

[45] H. Q. NGUYen AND B. PAusAder, A paradifferential approach for well-posedness of the Muskat problem, Arch. Ration. Mech. Anal., 237 (2020), pp. 35-100.

[46] C. W. Oseen, Sur les formules de Green généralisées qui se présentent dans l'hydrodynamique et sur quelquesunes de leurs applications, Acta Math., 35 (1912), pp. 97-192 .

[47] J. Simon, Compact sets in the space $L^{p}(0, T ; B)$, Ann. Mat. Pura Appl. (4), 146 (1987), pp. $65-96$.

Copyright (c) by SIAM. Unauthorized reproduction of this article is prohibited. 